\title{
Reticular dysgenesis-associated AK2 protects hematopoietic stem and progenitor cell development from oxidative stress
}

\author{
Alberto Rissone, ${ }^{1 *}$ Katja Gabriele Weinacht, ${ }^{7,9 *}$ Giancarlo la Marca, ${ }^{10,11}$ \\ Kevin Bishop, ${ }^{2}$ Elisa Giocaliere, ${ }^{11}$ Jayashree Jagadeesh, ${ }^{1}$ Kerstin Felgentreff, ${ }^{8}$ \\ Kerry Dobbs, ${ }^{8}$ Waleed Al-Herz, ${ }^{12,13}$ Marypat Jones, ${ }^{4}$ Settara Chandrasekharappa, ${ }^{4}$ \\ Martha Kirby, ${ }^{5}$ Stephen Wincovitch, ${ }^{6}$ Karen Lyn Simon, ${ }^{1}$ Yuval Itan, ${ }^{14}$ \\ Alex DeVine, ${ }^{7}$ Thorsten Schlaeger, ${ }^{7}$ Axel Schambach,, ${ }^{71}$ Raman Sood, ${ }^{2,3}$ \\ Luigi D. Notarangelo,, $16 * *$ and Fabio Candotti ${ }^{1,17 * *}$

\footnotetext{
'Disorders of Immunity Section, Genetics and Molecular Biology Branch; ${ }^{2 Z e b r a f i s h ~ C o r e ~ a n d ~}{ }^{3}$ Oncogenesis and Development Section, Translational and Functional Genomics Branch; ${ }^{4}$ Genomics Core, Cancer Genetics and Comparative Genomics Branch; ${ }^{5}$ Division of Intramural Research Flow Cytometry Core; and ${ }^{6}$ Cytogenetics and Microscopy Core, Genetic Disease Research Branch; National Human Genome Research Institute, National Institutes of Health, Bethesda, MD 20892

${ }^{7}$ Division of Hematology/Oncology and ${ }^{8}$ Division of Immunology, Boston Children's Hospital, Boston, MA 02115 ${ }^{9}$ Department of Pediatric Oncology, Dana-Farber Cancer Institute, Boston, MA 02115

${ }^{10}$ Department of Neurosciences, Psychology, Pharmacology, and Child Health, University of Florence, 51039 Florence, Italy

${ }^{11}$ Meyer Children's University Hospital, 50141 Florence, Italy

${ }^{12}$ Department of Pediatrics, Faculty of Medicine, Kuwait University, 13110 Kuwait City, Kuwait

${ }^{13}$ Allergy and Clinical Immunology Unit, Pediatric Department, Al-Sabah Hospital, 70459 Kuwait City, Kuwait

${ }^{14}$ St. Giles Laboratory of Human Genetics of Infectious Disease, Rockefeller Branch, The Rockefeller University, New York, NY 10065

${ }^{15}$ Institute of Experimental Hematology, Hannover Medical School, 30625 Hannover, Germany

${ }^{16}$ Harvard Stem Cell Institute, Harvard University, Cambridge, MA 02138

${ }^{17}$ Division of Immunology and Allergy, University Hospital of Lausanne, 1011 Lausanne, Switzerland
}

\section{CORRESPONDENCE \\ Fabio Candotti: \\ Fabio.Candotti@chuv.ch \\ OR \\ Luigi D. Notarangelo: \\ Luigi.Notarangelo@childrens. \\ harvard.edu}

Abbreviations used: AK, adenylate kinase; AP, alkaline phosphatase; ATRA, all-trans-retinoic acid; CHT, caudal hematopoietic tissue; DIG, digoxigenin; dpf, day postfertilization; dsRed, Discosoma sp. RFP; EB, embryoid body; EGFP, enhanced GFP; GSH, glutathione; hpf, hour postfertilization; HSPC, hematopoietic stem and progenitor cell; HSCT, hematopoietic stem cell transplantation; iPSC, induced pluripotent stem cell; ISH, in situ hybridization; MO, morpholino oligonucleotide; NAC, N-acetyl-L-cysteine; PFA, paraformaldehyde; RD, reticular dysgenesis; ROS, reactive oxygen species; RQ, relative quantification; WISH, whole mount ISH; ZFN, zinc-finger nuclease.

Adenylate kinases (AKs) are phosphotransferases that regulate the cellular adenine nucleotide composition and play a critical role in the energy homeostasis of all tissues. The AK2 isoenzyme is expressed in the mitochondrial intermembrane space and is mutated in reticular dysgenesis (RD), a rare form of severe combined immunodeficiency (SCID) in humans. RD is characterized by a maturation arrest in the myeloid and lymphoid lineages, leading to early onset, recurrent, and overwhelming infections. To gain insight into the pathophysiology of RD, we studied the effects of AK2 deficiency using the zebrafish model and induced pluripotent stem cells (iPSCs) derived from fibroblasts of an RD patient. In zebrafish, Ak2 deficiency affected hematopoietic stem and progenitor cell (HSPC) development with increased oxidative stress and apoptosis. AK2-deficient iPSCs recapitulated the characteristic myeloid maturation arrest at the promyelocyte stage and demonstrated an increased AMP/ADP ratio, indicative of an energy-depleted adenine nucleotide profile. Antioxidant treatment rescued the hematopoietic phenotypes in vivo in ak2 mutant zebrafish and restored differentiation of AK2-deficient iPSCs into mature granulocytes. Our results link hematopoietic cell fate in AK2 deficiency to cellular energy depletion and increased oxidative stress. This points to the potential use of antioxidants as a supportive therapeutic modality for patients with RD.

SCID includes a heterogeneous group of immunological diseases characterized by severe defects of T-lymphocyte number and function, variably

\footnotetext{
*A. Rissone and K.G. Weinacht contributed equally to this paper.

**L.D. Notarangelo and F. Candotti contributed equally to this paper.
}

associated with numerical and functional abnormalities of B and NK cells (Fischer et al., 2005; Aloj et al., 2012). Reticular dysgenesis (RD) is a
This article is distributed under the terms of an Attribution-Noncommercial-Share Alike-No Mirror Sites license for the first six months after the publication date (see http://www.rupress.org/terms). After six months it is available under a Creative Commons License (Attribution-Noncommercial-Share Alike 3.0 Unported license, as described at http://creativecommons.org/licenses/by-nc-sa/3.0/). 


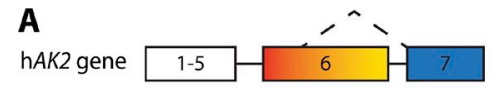

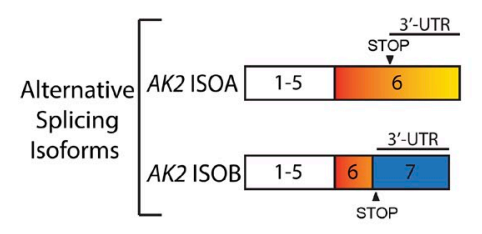

C zak2 gene
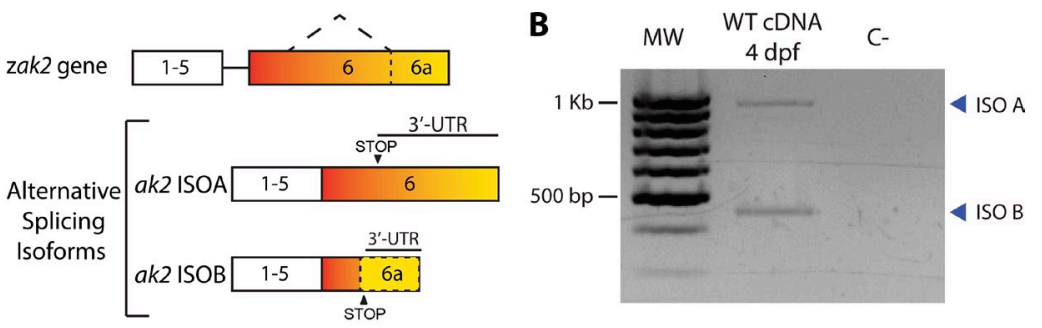

zAk2-ISOA MAPSTQEDDTVSGIRKGIRAILLGPPGAGKGTQAPKLAEKYCVCHLATGDMLRAMVASGSELGQRLKETMDAGKLVSDEMVVELIDNNLDTPACKNGFLLDGFPRTVKQAEMLDDLMEKRS 121 zAk2-ISOB MAPSTOEDDTVSGIRKGIRAILIGPPGAGKGTQAPKLAEKYCVCHLATGDMLRAMVASGSELGORLKETMDAGKLVSDEMVVELIDNNLDTPACKNGFLLDGFFRTVKOAEMLDDLMEKRS 121 hAK2-ISOA MAPSVPAAEPEY--PKGIRAVLLGPPGAGKGTQAPRLAENFCVCHLATGDMLRAMVASGSELGKKLKATMDAGKLVSDEMVVELIEKNLETPLCKNGFLLDGFPRTVRQAEMLDDLMEKRK 119 hAK2-ISOB MAPSVPAAEPEY--PKGIRAVLLGPPGAGKGTOAPRLAENECVCHLATGDMLRAMVASGSELGKKLKATMDAGKLVSDEMVVELIEKNLETPLCKNGELLDGEPRTVRQAEMLDDLMEKRK 119 ****. : $\quad . \quad * * * * *: * * * * * * * * * * * * *: * * *:: * * * * * * * * * * * * * * * * * * * * *:: * * * * * * * * * * * * * * * * * * *:: * * ; * * * * * * * * * * * * * * * *: * * * * * * * * * * * *$.

LID domain

z Ak2-ISOA EKLDSVIEFSVDDSLLVRRICGRLIHOPSGRSYHEEFHPPKEHMKDDVTGEPLIRRSDDNETTLRSRLESYHROTSPLVOYYSARGLHTAIDASQSTDLVFASILAAFSAATCKDLVYFV 241 $\mathrm{z}$ Ak2-ISOB EKLDSVIEFSVDDSLLVRRICGRLIHOPSGRSYHEEFHPPKEHMKDDVTGEPLIRRSDDNETTLRSRLESYHROTSPLVOYYSARGLHTAIDASOSTDLVFASILAAFSAATCV------- 235 h AK2-ISOA EKLDSVIEFSIPDSLLIRRITGRLIHPKSGRSYHEEFNPPKEPMKDDITGEPLIRRSDDNEKALKIRLOAYHTOTTPLIEYYRKRGIHSAIDASOTPDVVFASILAAFSKATCKDLVMFI 239 h AK2-ISOB EKLDSVIEFSIPDSLLIRRITGRLIHPKSGRSYHEEFNPPKEPMKDDITGEPLIRRSDDNEKALKIRLQAYHTQTTPLIEYYRKRGIHSAIDASQTPDVVFASILAAFSKATS-------- 232

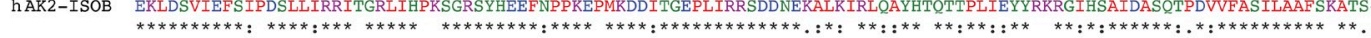

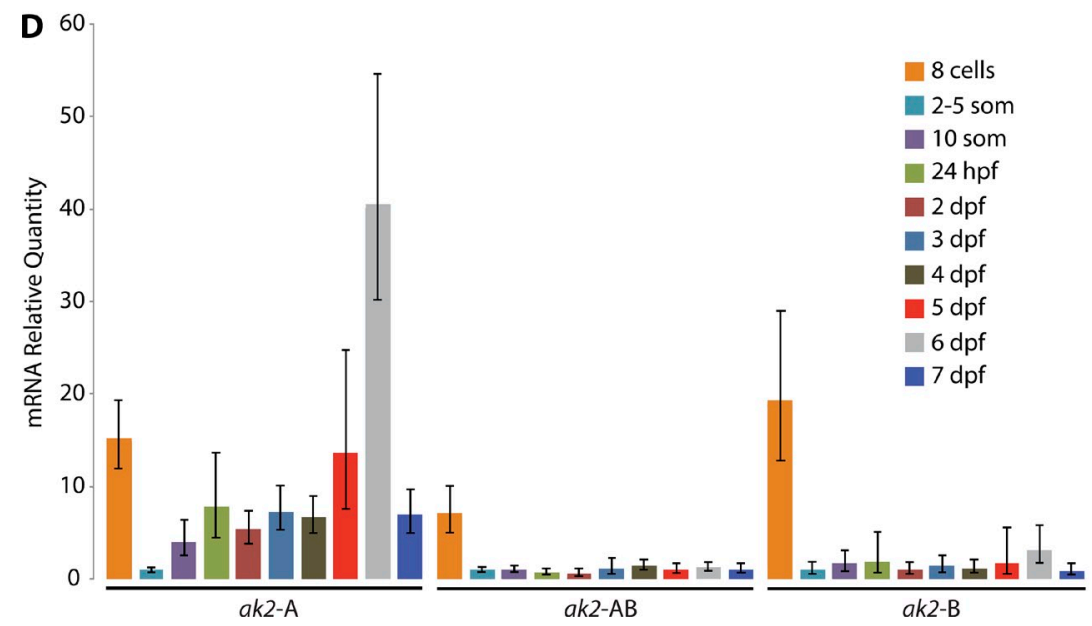

E $\quad a k 2$

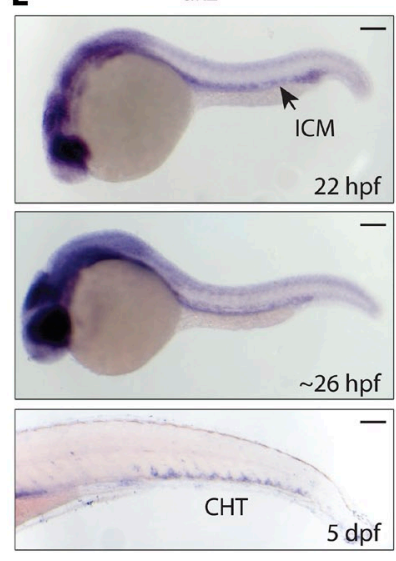

Figure 1. Zebrafish AK2 alternative splicing isoforms are expressed in hematopoietic regions during embryo development. (A) Schematic representation of human ( $\mathrm{A} A K 2$ ) and zebrafish (zak2) conserved gene structure and alternative splicing mechanism. STOP indicates the position of the STOP codons. Neither exons nor introns are drawn to scale. (B) RT-PCR analysis on cDNA of 4 dpf WT embryos. The reverse primer AK2-ISO B R2 (Table S1) was used in the PCR reaction to bind to isoforms A and B. MW, molecular weight size marker; $C-$, negative control. Blue arrowheads point to the two different alternative splicing isoforms. The results are representative of two independent experiments. (C) Multiprotein sequence alignment of human (hAK2) and zebrafish (zAk2) AK2 splicing isoforms (indicated as ISOA and ISOB). Features of human AK2 structure are depicted above the amino acid sequences. Different colors indicate different physicochemical properties. (D) mRNA quantitative real-time PCR analysis showing the relative expression of AK2 splicing isoforms during embryo development using specific probes for isoforms A or B ( $a$ k2-A and ak2-B) or a common probe for $A$ and $B$ isoforms (ak2-AB). Expression levels were normalized to $\beta$-actin 2 ( $\beta$-act 2 ) and elongation factor $1 \alpha$ (ef1 $\alpha$ ), and mRNA from 2-5-somite stage embryos was used as reference. Error bars indicate the calculated maximum $\left(R 0_{\max }\right)$ and minimum $\left(R 0_{\min }\right)$ expression levels that represent the standard error of the mean expression level (RO value). Data are pooled from at least three independent experiments. (E) Spatiotemporal analysis by stereomicroscopy of AK2 expression by WISH using a common probe for A and B isoforms at the indicated times. For each stage, at least 25 embryos have been analyzed. Data show one representative experiment out of two independently performed. ICM, intermediate cell mass; CHT, caudal hematopoietic tissue. Bars, $100 \mu \mathrm{m}$.

particularly severe, autosomal-recessive form of SCID and is characterized by virtual absence of circulating lymphocytes and neutrophils, as well as sensory neural hearing loss (LagreslePeyrou et al., 2009; Pannicke et al., 2009). The lack of innate and acquired immune function in patients with RD leads to extreme susceptibility to infections and early death, unless hematopoietic and immune reconstitution is achieved by allogeneic hematopoietic stem cell transplantation (HSCT). In 2009, two groups have independently reported that $\mathrm{RD}$ is caused by mutations in adenylate kinase 2 (AK2; Lagresle-Peyrou et al., 2009; Pannicke et al., 2009).

AKs are phosphotransferases that play an important role in cellular and mitochondrial energy homeostasis; moreover, they contribute to modulating the AMP-mediated response to stress signals (Dzeja and Terzic, 2003, 2009; Dzeja et al., 2007). Different AKs set the adenine nucleotide ratio in different intracellular compartments. In particular, AK2 is expressed in the mitochondrial intermembrane space where it regulates the 
concentration of mono- and diphosphorylated adenine nucleotides (Dzeja and Terzic, 2003, 2009; Dzeja et al., 2007). Through their distinct subcellular localization, AKs create a shuttle system to serve the needs of different cellular compartments for energetic and metabolic signaling; therefore, they represent a sensitive reporter of the cellular energy state (Dzeja and Terzic, 2003, 2009; Dzeja et al., 2007). Knockout studies have shown that the lack of a single AK enzyme is usually compensated at different levels through remodeling of the whole energetic network (Janssen et al., 2000; Pucar et al., 2000, 2002). For example, in $A k 1^{-/-}$mice, mitochondrial AK2 and AK3 can compensate at least in part for the lack of AK1 (Pucar et al., 2000). However, within the myeloid and lymphoid lineages, as well as in the stria vascularis of the inner ear, AK1 expression is absent or too low to compensate for the lack of AK2 activity in patients with RD (Le Guyader et al., 2008; Pannicke et al., 2009).

Since its first description (De Vaal and Seynhaeve, 1959), RD was assumed to be a disorder of primitive multipotent "reticular" progenitor cells primarily failing to develop into granulocytic and lymphoid precursors. However, the pathophysiology underlying the concurrent myeloid and lymphoid defects in RD has long remained unresolved.

To explore this topic, we studied the effects of AK2 deficiency in zebrafish embryos and human induced pluripotent stem cells (iPSCs) derived from an RD patient. Our results showed severe impairment of hematopoietic stem and progenitor cell (HSPC) development associated with increased oxidative stress and apoptosis in AK2-deficient zebrafish. Directed myeloid differentiation of AK2-deficient iPSCs resulted in the characteristic promyelocyte maturation arrest observed in RD patients and was associated with an energy-depleted adenine nucleotide profile. Notably, we found that treatment with antioxidants rescued the hematopoietic abnormalities in AK2 mutant zebrafish and restored granulocytic differentiation of AK2-deficient iPSCs. Our results support the hypothesis that AK2 deficiency results in energy depletion and abnormal redox state and suggest that antioxidants have potential as a supportive therapeutic modality in RD.

\section{RESULTS \\ AK2 deficiency induces a wide array of hematopoietic defects in zebrafish}

In humans, alternative splicing of the $A K 2$ gene can produce two different protein isoforms ( $\mathrm{A}$ and $\mathrm{B}$ ); isoform $\mathrm{B}$ is encoded by seven exons instead of six and has a different termination codon (Lee et al., 1998; Pannicke et al., 2009). One major characteristic of the zebrafish AK2 gene (ak2) is the lack of intron 6 , resulting in the juxtaposition of the exons 6 and 7 coding sequences (Fig. 1 A). Bioinformatic and RT-PCR analyses revealed a previously unreported isoform in zebrafish, corresponding to the human $A K 2$ isoform B. Although the zebrafish genomic sequence corresponding to the human $A K 2$ exon 7 represents part of the $3^{\prime}$-UTR region of isoform A, RT-PCR and sequence alignment analyses indicated that alternative splicing mechanisms are conserved (Fig. 1, A-C).
To assess the characteristics of physiological ak2 expression during zebrafish development, we performed quantitative real-time PCR and whole mount in situ hybridization (ISH [WISH]) experiments. The quantitative real-time PCR analysis showed that zebrafish embryos inherit both maternal $A$ and $B$ transcripts; moreover, relative quantification (RQ) using the 2-5-somite stage as reference indicated a significant decline in transcription levels after the beginning of zygotic transcription (Fig. 1 D). WISH experiments at different stages of development using a common ISH probe for isoforms A and B (Fig. 1 E) localized ak2 expression to hematopoietic regions such as the intermediate cell mass and the caudal hematopoietic tissue (CHT) around $24 \mathrm{~h}$ postfertilization (hpf) and $5 \mathrm{~d}$ postfertilization (dpf), respectively.

No mouse model for AK2 deficiency has been reported in the literature to date, and mouse lines carrying homozygous ak2-inactivating retroviral insertions are embryonically lethal (unpublished data). To study the role of AK2 in hematopoietic development, we set out to generate a zebrafish model of AK2 deficiency. Because RD patients present with a variety of different mutations (including large intragenic deletions, single nucleotide substitution, and frameshift mutations; LagreslePeyrou et al., 2009, 2011; Pannicke et al., 2009; Al-Zahrani et al., 2013; Henderson et al., 2013), we decided to generate various ak2 zebrafish mutants. Using zinc-finger nuclease (ZFN) technology, we introduced frameshift mutations in the first exon of the ak2 gene (a 2-bp deletion and a 4-bp insertion, indicated as $a k 2^{\text {del } 2}$ and $a k 2^{i n s} 4$, respectively; Fig. 2 A; Sood et al., 2013). In addition, we screened a zebrafish DNA library of ENU-induced mutations (Sood et al., 2006) and uncovered a mutant fish line carrying a T371C/L124P missense mutation within exon 4 of the AK2 gene. The mutated amino acid in this mutant (Fig. 2 B, red arrows) falls in a highly conserved region of AK2 (Liu et al., 2009) located upstream of the $\beta$ sheet connecting the CORE domain to the LID domain. The L124P mutation has a PROVEAN score (Choi et al., 2012) equal to -6.9 and was predicted to affect protein stability and enzymatic activity. Genotyping of in-crossed heterozygous embryos confirmed the presence of these recessive mutations in a normal Mendelian ratio (Fig. $2 \mathrm{~B}$, right). Contrary to the ZFN-induced frameshift mutations that resulted in abrogation of ak2 mRNA, the L124P substitution did not affect production of the mRNA transcript (Fig. 2 C).

A previous study has shown that morpholino oligonucleotide (MO)-mediated knockdown of ak2 expression induced defects in zebrafish leukocyte development (Pannicke et al., 2009). We confirmed and refined these findings in our zebrafish ak2 mutant lines that showed profound impairment of lymphoid and myeloid development, as indicated by lack of ikaros and rag 1 expression in the thymic anlage and defective $m p x$ expression at $5 \mathrm{dpf}$ (Fig. $2 \mathrm{D}$ ). Because RD is characterized by agranulocytosis, we used Sudan black, a lipid stain which labels the granules of granulocytes (Sheehan and Storey, 1947; Le Guyader et al., 2008), to visualize mature granulocytes in zebrafish larvae. When comparing Sudan black-stained AK2 mutants to control embryos, we found a progressive 
A
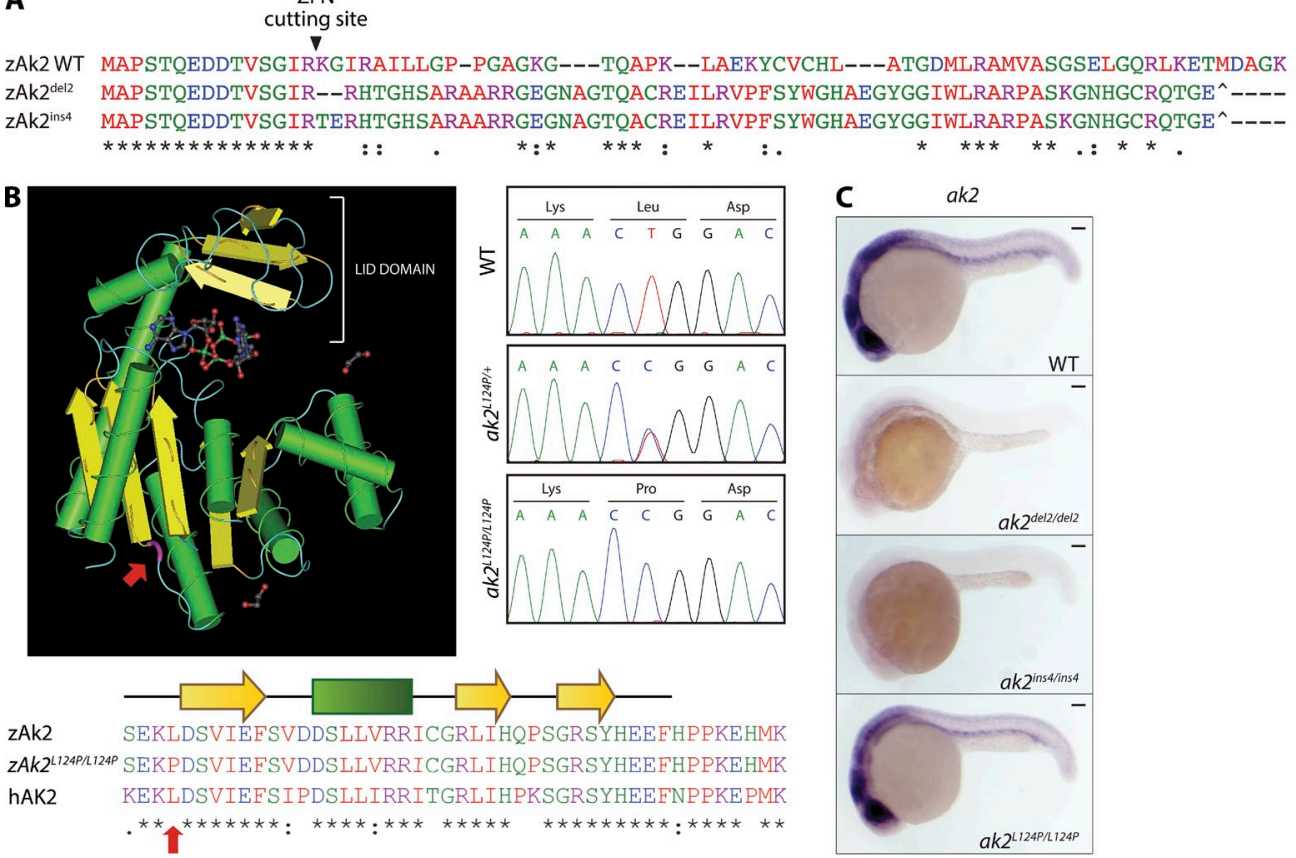

D

WT

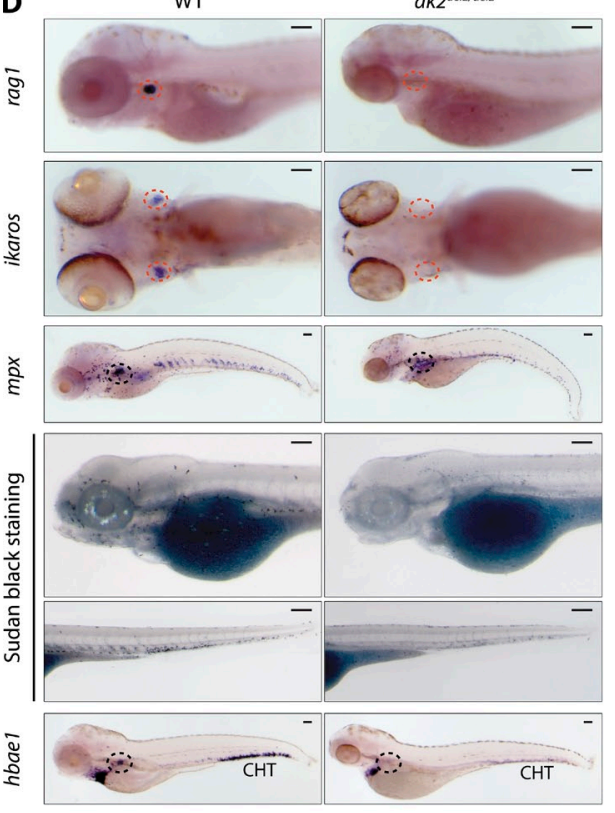

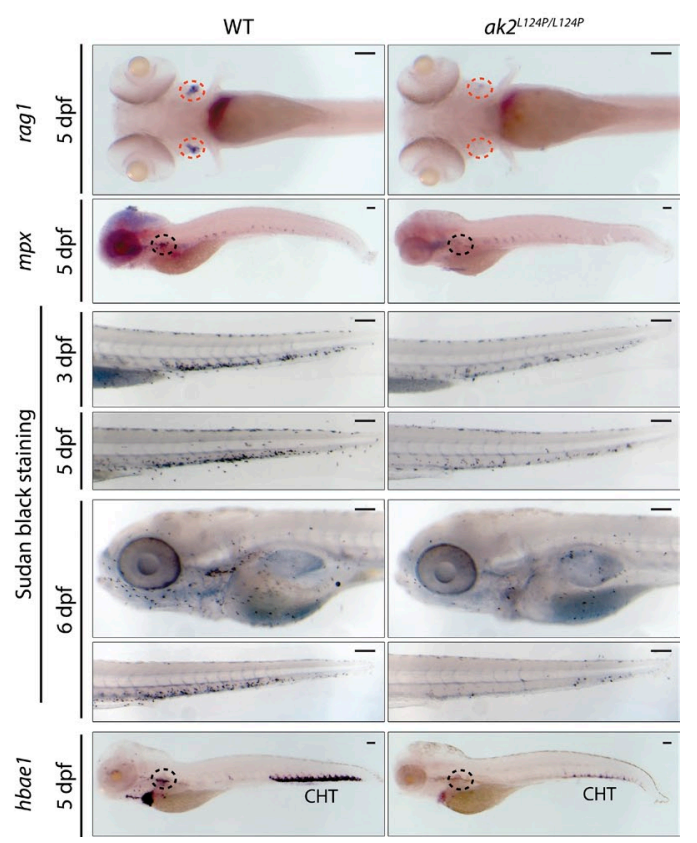

Figure 2. Zebrafish AK2 mutants present a wide array of hematopoietic defects. (A) Alignment of protein sequences encoded by the WT and ZFN-induced mutant alleles (del2 and ins4) shows the predicted frameshift after the Arg 15 codon targeted by the ZFN cutting site. Premature stop codons are also indicated (^). (B) 3D structure of human AK2 (left). (bottom) Partial sequence multi-alignment of WT (zAk2) and missense mutant zebrafish Ak2 (zAk2 ${ }^{L 124 P / L 124 P}$ ) and human AK2 (hAK2). The mutated amino acid position is highlighted in both panels (red arrows). (right) Sequence chromatograms showing the nucleotide change for L124P missense mutation in heterozygous ( $a k^{L 124 P /+}$ ) and homozygous ( $a k 2^{L 124 P / L 124 P}$ ) embryos compared with the WT sequence. (C) Stereomicroscope images of WISH analysis on $24 \mathrm{hpf} \mathrm{WT} \mathrm{and} \mathrm{mutant} \mathrm{embryos} \mathrm{using} \mathrm{an} \mathrm{antisense} \mathrm{probe} \mathrm{against} \mathrm{ak2} \mathrm{mRNA} \mathrm{(A} \mathrm{and} \mathrm{B} \mathrm{tran-}$ scripts). For each sample, at least 25 embryos have been analyzed. (D) Analysis of definitive hematopoietic phenotypes in $a k 2^{d e l / 2 / 2 / 2}$ and $a k 2^{L 124 P / L 124 P}$ mutants by WISH and stereomicroscopy. Red and black dashed circles indicate thymus and kidney regions, respectively. (left) Lateral views of embryos hybridized with rag 1, mpx, and hbae 1 and ventral views of ikaros hybridization at $5 \mathrm{dpf}$. Lateral views of embryos stained using Sudan black solution to mark granulocytes at $5 \mathrm{dpf}$. (right) Ventral views of rag 1 and lateral views of mpx and hbae 1 hybridized embryos at 5 dpf and lateral views of embryos stained using Sudan black solution at different stages of development. For each sample, at least 25 embryos have been analyzed. Data in $C$ and $D$ show one representative experiment out of two independently performed. Bars, $100 \mu \mathrm{m}$. 

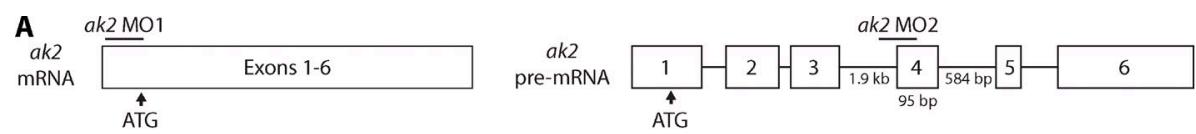

B

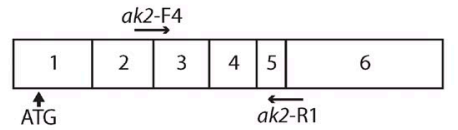

Predicted ak2 transcript including exon 4 (exon4+)
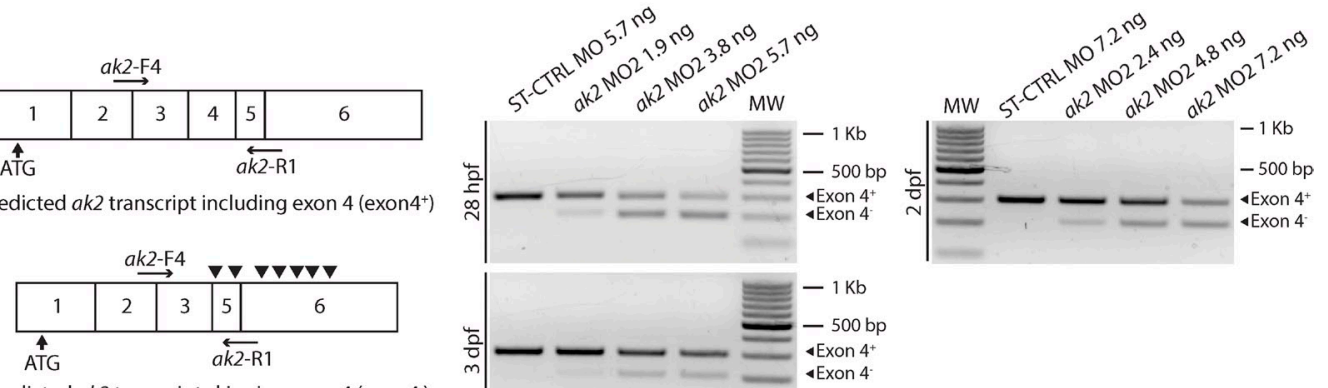

Predicted ak2 transcript skipping exon 4 (exon4)
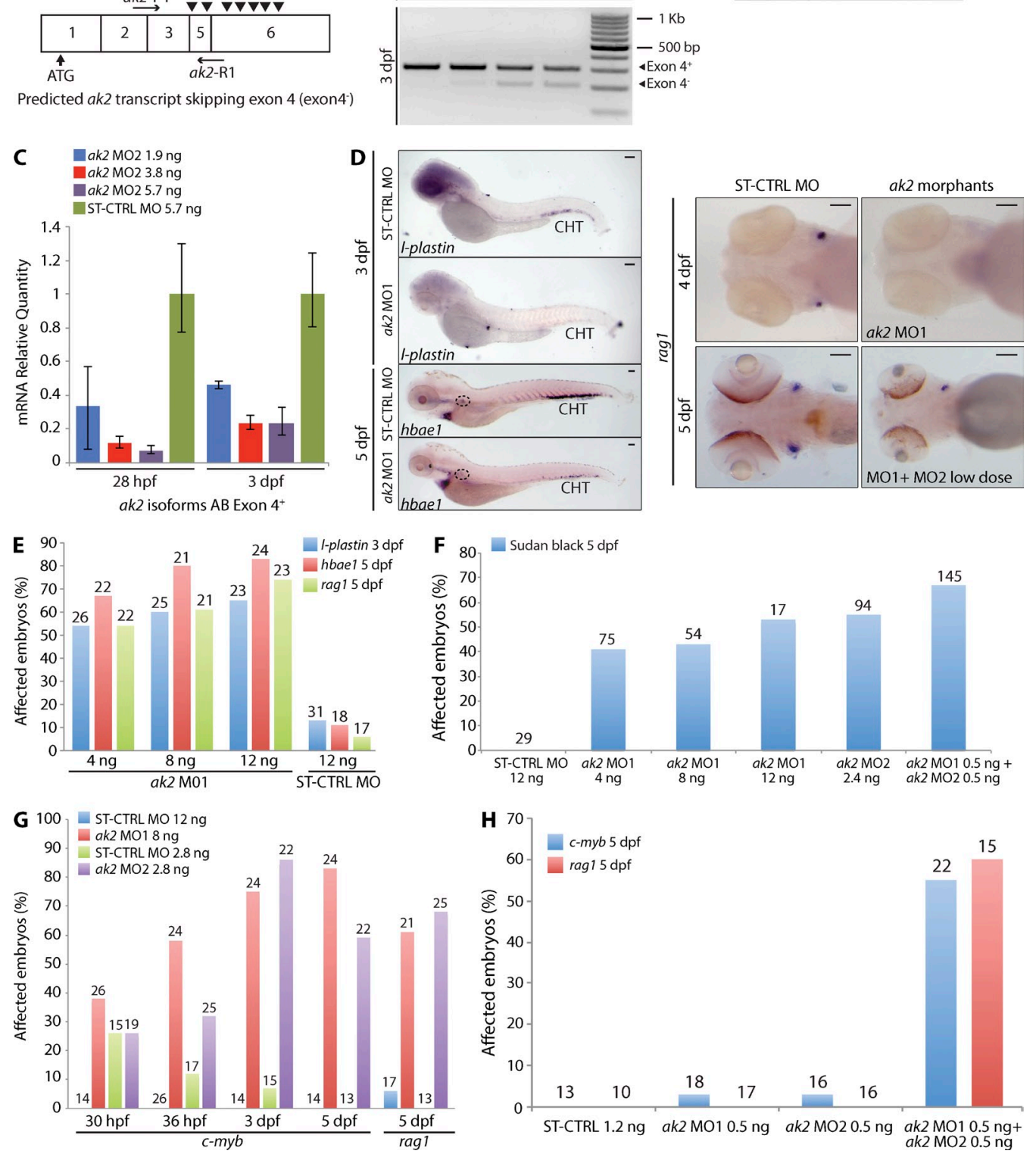

Figure 3. ak2 knockdown by MO injection phenocopies hematopoietic defects observed in ak2 zebrafish mutants. (A) Schematic representation of the two different MOs used in the study: ak2 M01 blocking the mRNA translation and ak2 M02 targeting the intron 3-exon 4 splice junction of the ak2 gene. Neither exons nor introns are drawn to scale. Numbers below the introns (straight lines) and exons (white boxes) indicate their size in bases. (B) Schematic representation of the predicted AK2 transcripts in MO-treated embryos. Black arrowheads in the bottom left panel indicate noncanonical STOP codons introduced by ak2 M02-induced skipping of exon 4. Horizontal black arrows indicate the pair of primers used in RT-PCR (right panels) to test the effects of different doses of ak2 M02 during development as indicated. MW, molecular weight size marker. (C) mRNA quantitative real-time PCR analysis of splicing MO activity (three different doses) at $28 \mathrm{hpf}$ and $3 \mathrm{dpf}$. In both cases, ST-CTRL MO-injected embryos serve as reference. The error bars indicate the calculated maximum $\left(\mathrm{RO}_{\text {max }}\right)$ and minimum $\left(\mathrm{RO}_{\min }\right)$ expression levels that represent the standard error of the mean expression levels 
reduction in Sudan black-positive cells throughout the larva bodies (Fig. 2 D). Interestingly, we also observed markedly impaired expression of the erythroid marker hbae1 (Fig. 2 D). These results were confirmed by injecting translation-blocking (ak2 MO1) or aberrant splicing-inducing (ak2 MO2) MOs (Pannicke et al., 2009), either individually or in combination (Fig. 3). The expected effects of the splice-blocking MO injection (ak2-MO2, targeting intron 3-exon 4 of AK2 premRNAs) included the skipping of exon 4 and a frameshift resulting in the introduction of multiple STOP codons (Fig. 3, A and B). RT-PCR and quantitative real-time PCR analyses confirmed the skipping of exon 4 and indicated that the spliceblocking $\mathrm{MO}$ was still active at 3-dpf stage, although with reduced activity (Fig. 3, B and C). The findings of impaired lymphoid and myeloid development in both AK2 mutant and ak2 MO-injected (morphant) zebrafish embryos (Fig. 3, D-F) strongly indicate that the observed hematopoietic phenotype represents a direct consequence of AK2 deficiency.

\section{AK2 deficiency affects zebrafish HSPC development}

Because AK2 deficiency in zebrafish resulted in developmental defects of all hematopoietic lineages, we hypothesized that HSPC development might also be affected. Analysis of c-myb and runx 1 expression in hematopoietic tissues revealed only minor differences between WT and AK2 mutants or morphants during early phases of HSPC development (i.e., 30-36 hpf; Fig. 4, A, B, and D). Moreover, WISH analysis with a panendothelial vascular marker $(c d h 5)$ and confocal analysis on embryos from incross of $a k 2^{\text {del2/+ }}$ mutants in the $\mathrm{Tg}$ (fli1a: EGFP) $)^{r^{1}} ; \mathrm{Tg}$ (gata1a:dsRed $)^{d 2}$ background indicated a correct vascular development (marked by enhanced GFP [EGFP] expression) and the presence of active circulation (marked by Discosoma sp. RFP [dsRed] expression) in mutant embryos from $24 \mathrm{hpf}$ to $2 \mathrm{dpf}$ (Fig. 4 E). Interestingly, however, both AK2 mutants and morphants showed a strong reduction of c-myb expression at 2 dpf through 5 dpf (Fig. 3, G and H; and Fig. 4, A-C).

To obtain additional evidence that HSPC development was in fact impaired, we injected $T g(c d 41: G F P)$ embryos with the ak2 MOs, either separately or in combination at low dose (Fig. 4, F and G). In this specific transgenic line, HSPCs and thrombocytes are marked by GFP expression (Lin et al., 2005; Bertrand et al., 2008; Kissa et al., 2008; Renaud et al., 2011). At $4.5 \mathrm{dpf}$, control embryos displayed $\mathrm{GFP}^{+}$cells (HSPCs and thrombocytes) in the CHT region and in the circulation. However, consistent with the aforementioned described findings, $\mathrm{Tg}(c d 41$ :GFP)/AK2 morphant embryos showed strong reduction of $\mathrm{GFP}^{+}$cells in the CHT region (Fig. 4, F and G). Collectively, these data support an important role for AK2 in HSPC development during zebrafish definitive hematopoiesis.

\section{AK2 deficiency increases oxidative stress and apoptosis in zebrafish hematopoietic tissues}

Under physiological conditions, the amount of oxidative stress produced in the mitochondria is counteracted by an intricate defense system that scavenges and detoxifies reactive oxygen species (ROS). Mitochondrial dysfunction can lead to excess ROS production that exhausts the cellular antioxidant capacity. Unopposed oxidative stress leads to apoptosis (Craven et al., 2005). Attempts to compensate for increased levels of ROS include activation of the expression of different genes such as the inducible isoform of heme oxygenase (HMOX1; Ryter and Choi, 2002). Therefore, HMOX1 expression serves as a marker of increased levels of cellular oxidative stress. We reasoned that the reduced ADP recycling caused by AK2 deficiency would result in decreased ATP production, mitochondrial dysfunction, and increased ROS production. Indeed, CHT cells from ak2 $2^{\text {ins } 4 \text { ins } 4}$ and $a k 2^{\text {L124P/L124P }}$ embryos showed evidence of oxidative stress as detected by the MitoSOX and CellROX indicators (Fig. $5 \mathrm{~A}$ and not depicted). In addition, FACS analysis of WT and ak2del/del 2 embryo-derived cells stained with MitoSOX showed significantly increased levels of superoxide in mitochondria of null mutant cells at 4 and $5 \mathrm{dpf}$, with similar levels of apoptotic and dead cells as indicated by Annexin V-APC and 7-AAD staining, respectively (Fig. 5, B and C). CellROX staining at $4 \mathrm{dpf}$ confirmed the presence of higher levels of oxidative stress in null mutant embryo cells (Fig. 5 D), and hmox $1 a$ expression in the CHT of both ak2del/del2 and ak2 $2^{L 124 P / L 124 P}$ mutant embryos showed dramatic induction starting at $2 \mathrm{dpf}$, in contrast to WT control embryos that showed very few or no hmox 1a-positive cells in the same region (Fig. 5, $\mathrm{E}$ and $\mathrm{F})$. Analogous to what is observed in human fibroblasts derived from individuals with RD (Pannicke et al., 2009), these results demonstrate that, in zebrafish, AK2 deficiency induces oxidative stress in hematopoietic tissues.

Previous reports have linked decreased AK2 activity to induction of apoptosis (Pannicke et al., 2009; Burkart et al.,

(RO value). (D-H) Qualitative and quantitative analysis of hematopoietic defects induced by ak2 MOs injection during definitive hematopoiesis assessed by WISH and stereomicroscopy. WT Ekkwill (EK) embryos were injected with different doses (4, 8, and $12 \mathrm{ng}$ ) of ak2 M01 or ak2 M02 (2.4 ng) and analyzed by WISH at different developmental stages using specific hematopoietic probes. ST-CTRL MO at the highest dose used for ak2 M01 was used as a control. Numbers above bars indicate the total count of embryos analyzed. (D, left) Stereomicroscope lateral views of I-plastin (marker of macrophages and monocytes) and hbae 1 WISH signals in ST-CTRL and ak2 M01 morphants at 3 and $5 \mathrm{dpf}$. Black dashed circles indicate the position of the kidney. (right) Stereomicroscope ventral views of ST-CTRL MO, ak2 MO1 morphants, or ak2 M01- and M02-coinjected embryos hybridized with rag 1 ISH probe to visualize differentiating lymphocytes in the thymus. For each sample, at least 25 embryos have been analyzed. Bars, $100 \mu \mathrm{m}$. (E) Effect of ak2 M01 and ST-CTRL MO injection on the expression of I-plastin, hbae1, and rag1. (F) Effect of single ak2 M0 injection and low dose coinjection on granulopoiesis (Sudan black stain). ( $\mathrm{G}$ and $\mathrm{H}$ ) Effect of single ak2 MO injection or low dose coinjection on the expression of c-myb and rag 1 from $30 \mathrm{hpf}$ to $5 \mathrm{dpf} .$. Data are presented as percentage of affected embryos. All data $(\mathrm{B}-\mathrm{H})$ show one representative experiment out of at least two independently performed. 

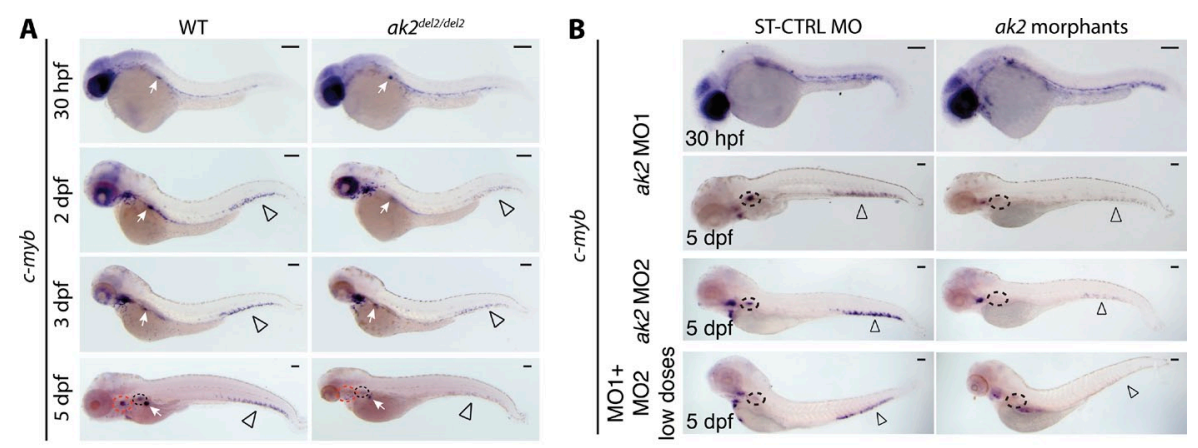

Figure 4. AK2 deficiency impairs HSPC development in zebrafish. (A-C) Representative stereomicroscope images of WISH analyses of c-myb expression in $a k 2^{\text {del/2/de/2 null }}$ mutants, ak2 morphants, and ak2 L124P/L124P mutants. Red and black dashed circles mark thymus and kidney regions, respectively; open arrowheads indicate $\mathrm{CHT}$ regions. (A) Lateral views of right side of mutant embryos at different developmental stages compared with their WT siblings. White arrows mark insulin expression (positive control). (B) Lateral views
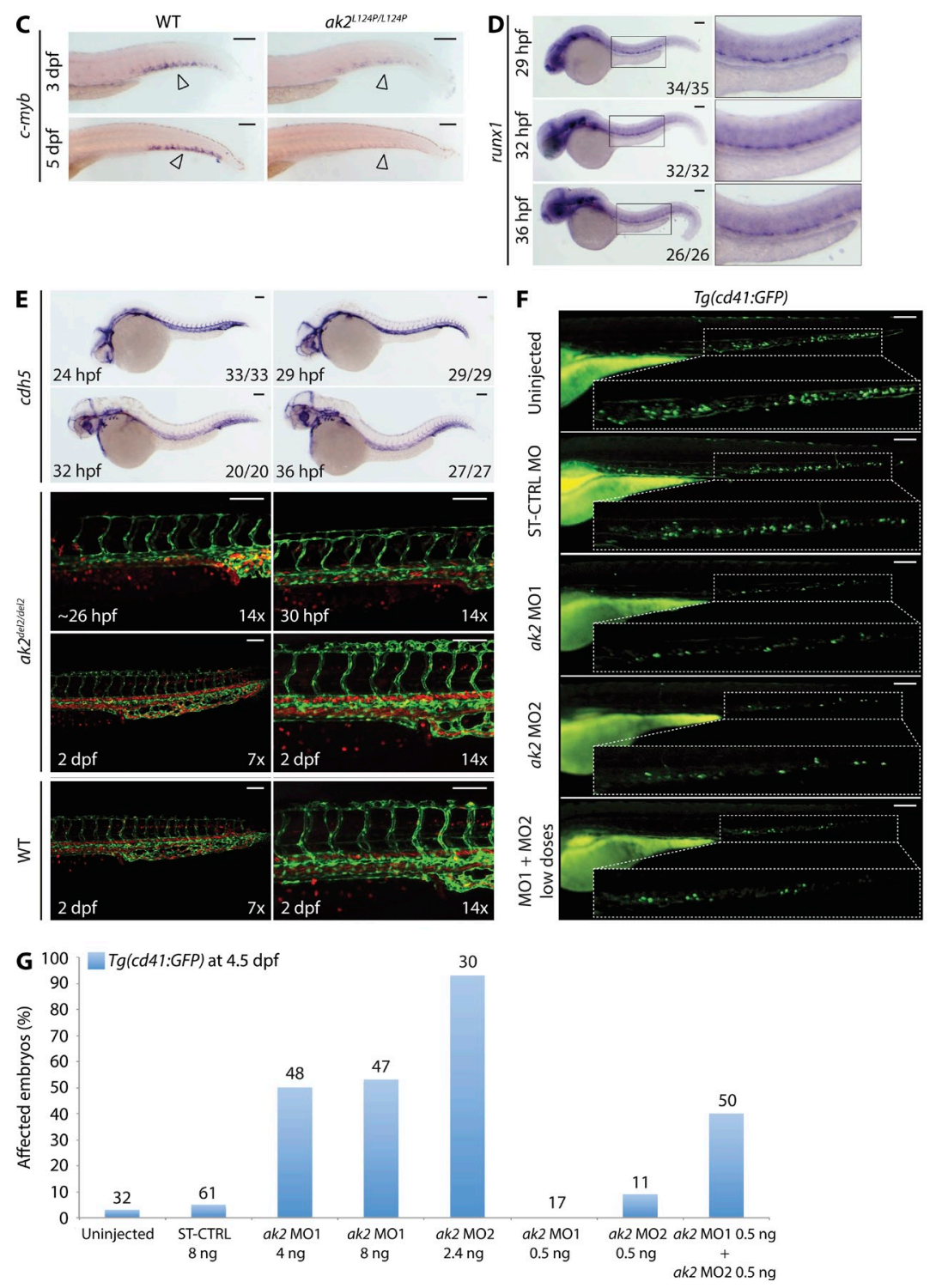
of ST-CTRL MO and ak2 MO morphants at different developmental stages as indicated. Two different ak2 MOs (M01 or M02) were injected separately or in combination at low dose (M01 + M02 low doses). (C) Lateral views of the trunk/tail regions of ak2 $2^{L 24 P / L 124 P}$ mutant embryos at 3 and $5 \mathrm{dpf}$ compared with their WT siblings. (D) Stereomicroscope images of WISH analysis of runx 1 expression from 29 to $36 \mathrm{hpf}$ in ak2 del2 embryos from an intercross of heterozygous ( $a k^{\text {de/2/+ }}$ ) adults $(35,32$, and 26 embryos analyzed at 29, 32 , and $36 \mathrm{hpf}$, respectively). (E) Analysis of vascular system development from 24 to $48 \mathrm{hpf}$ in ak $2^{d e / 2}$ mutant embryos. (top) Stereomicroscope lateral views of WISH analysis with a cdh5 antisense probe on embryos from an intercross of heterozygous ( $a k 2^{\text {del/2/+ })}$ adults $(33,29,20$, and 27 embryos analyzed at 24, 29,32 , and 36 hpf, respectively). (bottom) Confocal microscopy lateral views of the trunk/tail regions of ak2 $2^{\text {del/del } / 2}$ double transgenic $T g(f l i 1 a: E G F P){ }^{1}$; Tg (gata 1a:dsRed)sd2 embryos and WT controls at different developmental stages; fli1a (EGFP) marks vascular development, and gata-1 (dsRed) marks red blood cells as indicated. (F) Stereomicroscope lateral views of trunk/tail region of $4.5 \mathrm{dpf}$ $\mathrm{Tg}$ (cd41:GFP) embryos injected with different ak2 MOs. (G) Quantitative analysis of ak2 MO injections on $\mathrm{Tg}$ (cd41:GFP) embryos. Numbers above bars indicate the total number of embryos analyzed. Data are presented as percentage of affected embryos. For each panel $(A-G)$, results of one representative experiment out of at least two independent replicates are shown. (A-F) For each sample at least, 20 embryos have been analyzed. Bars, $100 \mu \mathrm{m}$.

2011; Henderson et al., 2013). To test this hypothesis, we assayed for cell death using acridine orange staining and TUNEL assay. We detected apoptotic cells in the CHT of ak2 morphants at $3 \mathrm{dpf}$ (Fig. $5 \mathrm{G}$ ) and confirmed the increased number of apoptotic cells by TUNEL assay in CHT regions of $a k 2^{\text {del2/del2 }}$ and $a k 2^{L 124 P / L 124 P}$ mutant embryos (Fig. 5, H and I).
These data suggest that the progressive reduction of HSPCs and committed hematopoietic precursors in AK2-deficient embryos can be attributed to increased levels of ROS, resulting in cell death by apoptosis, which is in line with observations made in AK2-deficient in vitro model systems (Lagresle-Peyrou et al., 2009; Pannicke et al., 2009). 

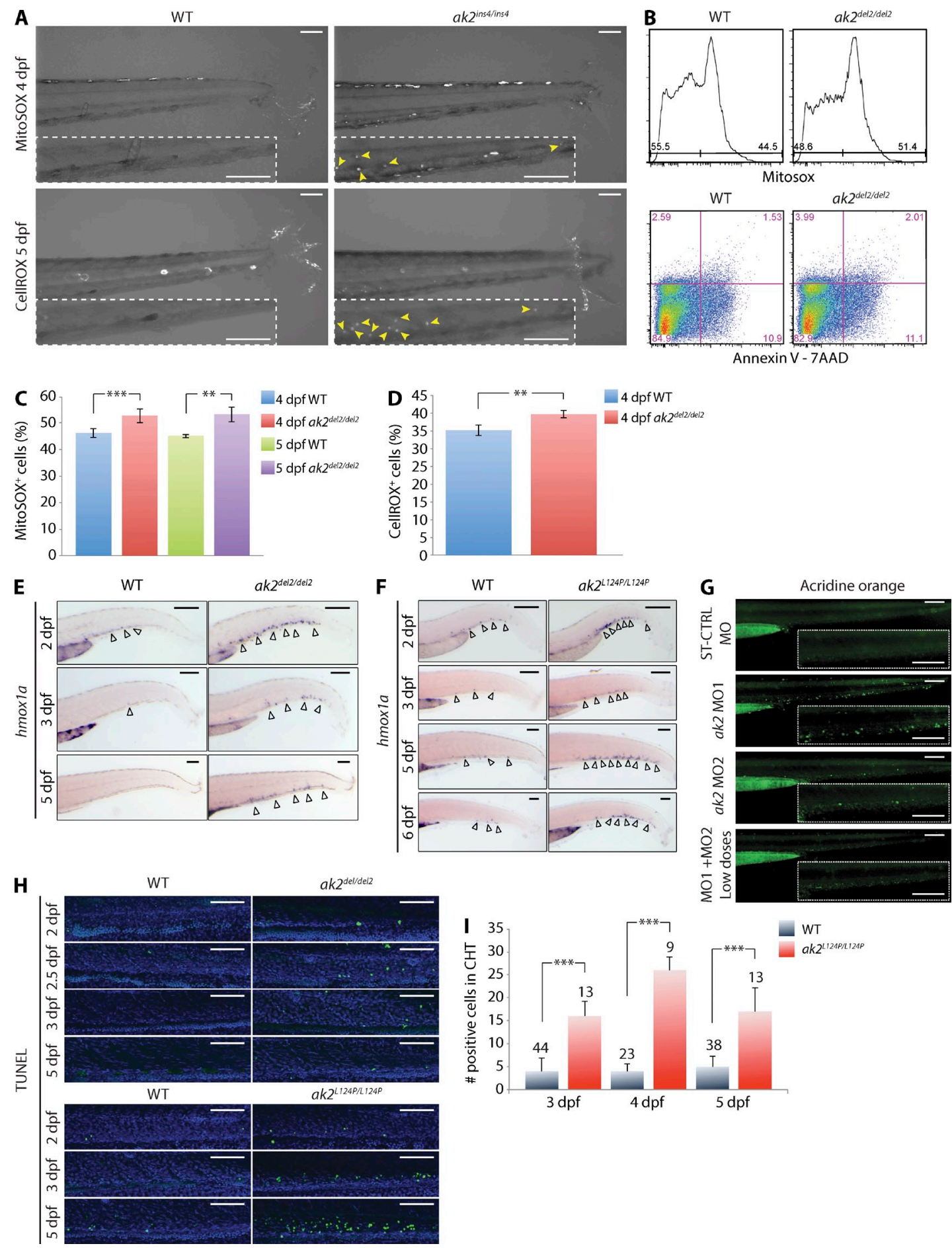

Figure 5. AK2 mutant zebrafish demonstrate increased levels of cellular oxidative stress and apoptosis in hematopoietic tissues. (A) Confocal microscopy assessment of oxidative stress in the CHT region of WT and ak2ins4/inst mutant embryos probed with the MitoSOX or CellROX indicators at the indicated stages. Yellow arrowheads in the dashed insets indicate MitoSOX- or CelIROX-positive cells in CHT regions. (B) Representative quantitative analysis by flow cytometry of oxidative stress, apoptosis, and cell death on $5 \mathrm{dpf}$ ak2 del/del2 and control embryos (60 embryos each) using MitoSOX Red and Annexin V + 7-AAD, respectively. (C and D) Quantitative analysis of MitoSOX Red (C) and CellROX Green (D) staining at 4 and $5 \mathrm{dpf}$ in ak2del/del2 mutants and WT siblings (60 embryos each). (E) WISH analysis of hmox 1a expression in ak2 del/del2 mutants and their WT siblings at different stages of development. (F) WISH analysis of hmox1a expression in ak2 ${ }^{124 P / L 124 P}$ mutants and their WT siblings at different stages of development. (E and F) Open arrowheads indicate hmox1a-positive cells. (G) Stereomicroscope analysis of acridine orange staining of AK2 morphants at 3 dpf. Dashed insets show CHT

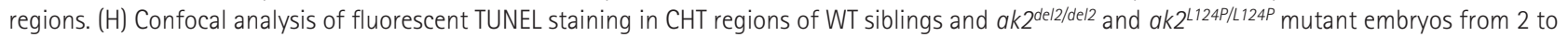
$5 \mathrm{dpf}$. Each panel represents a crop of the CHT region from a 14x magnification image. The green signal indicates TUNEL-positive cells and the blue signal 


\section{Antioxidant treatment rescues hematopoietic phenotypes in AK2-deficient embryos}

To further corroborate our findings, we investigated the effects of different antioxidant compounds on oxidative stress and hematopoietic abnormalities noted in ak2 mutant zebrafish. We exposed an unselected population of embryos derived from intercross of $a k 2^{L 124 P}$ heterozygous missense mutants to different concentrations of $N$-acetyl-L-cysteine (NAC). This treatment resulted in decreased cellular oxidative stress, as indicated by a $50 \%$ reduction of $h m o x 1 a$ induction in NAC-treated $a k 2^{L 124 P / L 124 P}$ missense mutant zebrafish versus untreated mutants (Fig. 6 A). Moreover, antioxidant treatment rescued the defective expression of $\mathrm{c}-m y b$, hbae 1 , and rag 1 in $\sim 50 \%$ of the embryos expected to show an abnormal phenotype (Fig. 6 A, left).

Importantly, similar results were observed for c-myb and rag1 expression after treatment of ak $2^{d e l 2 / d e l 2}$-null mutant embryos with different concentrations of NAC, glutathione (GSH), and GSH reduced ethyl ester (GSH-MEE), a membrane/lipidpermeable derivative of GSH (Fig. 6, B-E). Notably, we did not observe macroscopic defects, nor enhanced expression of hematopoietic markers in WT embryos treated with antioxidants. Overall, these data indicate that antioxidant treatment ameliorates the hematopoietic phenotypes observed in zebrafish ak2 mutants and suggest a crucial role of oxidative stress in the pathogenesis of RD.

\section{Patient-derived iPSCs as an in vitro model for RD}

To assess whether our results in zebrafish may be translated into new therapeutic approaches for patients with RD, we developed a disease model based on in vitro hematopoietic differentiation of iPSCs. Dermal skin fibroblasts were derived from a patient who presented with agranulocytosis, severe $\mathrm{T}$ cell lymphopenia, and absent brain stem auditory-evoked response at birth, the characteristic triad that clinically defines RD. The patient was found to carry a homozygous point mutation (c.524G>A) in exon 6 of the $A K 2$ gene, leading to an arginine to glutamine substitution (p.R175Q) in the LID domain of the AK2 protein (Fig. 7, A and B; Henderson et al., 2013). Western blot analysis from patient fibroblasts and $\mathrm{CD}^{+} \mathrm{T}$ cells demonstrated reduced protein expression compared with control (Henderson et al., 2013), and a PROVEAN score of -3.871 predicted this mutation to be deleterious. The cultured fibroblasts were reprogrammed to pluripotency by using an excisable, self-inactivating, codon-optimized lentiviral vector carrying OCT4, SOX2, KLF4, $c M Y C$, and the reporter dTomato in a single cassette (Warlich et al., 2011). Immunofluorescence imaging and quantitative realtime PCR analysis confirmed the stemness of the generated iPSC line by expression of the characteristic pluripotency markers TRA-1-81, TRA-1-60, NANOG, OCT-4, SSEA-3, and SSEA-4 (Fig. 7, C and D). Karyotype and G-banding analyses demonstrated cytogenetic integrity, and gDNA sequencing showed genotypic identity with the parental fibroblast line (Fig. 7, E and F). Of note, the reprogramming efficiency of $0.0096 \%$ in AK2-deficient fibroblasts (mean over eight experiments) was $\sim 500$-fold lower than the reprogramming efficiency of $4.75 \%$ seen in the WT control (mean over three experiments).

\section{AK2 deficiency affects in vitro granulopoiesis and erythropoiesis}

To model the hematopoietic phenotype of RD in vitro, patientderived iPSCs were grown in suspension culture to form embryoid bodies (EBs), dissociated into single cells, and plated in a CFU assay in the presence of SCF, FLT3, BMP4, G-CSF, IL3, and IL6. AK2 2175 Q/R175Q iPSCs recapitulated the characteristic maturation arrest of the myeloid lineage at the promyelocyte stage that is observed in the bone marrow of RD patients (Lagresle-Peyrou et al., 2009; Pannicke et al., 2009), whereas the control demonstrated differentiation into mature neutrophils with distinctive nuclear segmentation (Fig. $8 \mathrm{~A}$ ). For unequivocal delineation of the differentiation stage, we performed electron microscopy on AK2 R175Q/R175Q iPSCderived myeloid precursors and control cells. Cells morphologically identified as promyelocytes by light microscopy displayed abundant electron-dense primary granules, validating their promyelocyte nature (Fig. 8 B). In addition to the maturation arrest, the quantitative potential of AK2-deficient cells to form mixed myeloid lineage colonies was significantly decreased (Fig. 8 C). Interestingly, we also noted abnormalities in the erythroid differentiation of RD iPSCs. Red cell precursors derived from $\mathrm{AK} 2{ }^{\mathrm{R} 175 \mathrm{Q} / \mathrm{R} 175 \mathrm{Q}}$ iPSCs displayed obvious dysmorphic features such as bilobate nuclei and inconsistent nucleus-to-cytoplasm ratio (Fig. $8 \mathrm{D}$ ), and their potential to give rise to CFUserythroid (CFU-E) and burst-forming units-erythroid (BFU-E) was severely compromised (Fig. 8 E). The impact of AK2 deficiency on more than one hematopoietic cell lineage in the iPSC model supports our findings in the zebrafish, suggesting that RD affects HSPC development and differentiation.

AK2 deficiency affects the cellular adenine nucleotide profile AK2 is considered the primary mitochondrial ADP generator (Dzeja and Terzic, 2009). We therefore reasoned that decreased AK2 activity would alter the cellular balance between AMP and ADP toward a relative increase in AMP. Using tandem mass spectrometry, we quantified the intracellular nucleotide content. In AK2 $2^{\text {175Q/R175Q_derived myeloid cells, the AMP/ }}$ ADP ratio was markedly skewed toward AMP, whereas intracellular ADP levels were decreased (Fig. 8 F). These data show that AK2 deficiency results in decreased cellular ADP supply, which may limit substrate availability for the ATP synthase and compromise cellular respiration.

indicates DAPI staining. (A and E-H) For each sample, at least 25 embryos have been analyzed. Bars, $100 \mu \mathrm{m}$. (I) Quantitative analysis of TUNEL AP staining on WT siblings and $a k 2^{L 124 P / L 124 P}$ missense mutant embryos from 3 to $5 \mathrm{dpf}$. The number of embryos analyzed is shown above each column. In panels $C, D$, and I, error bars indicate standard deviation; ${ }^{* *}, P<0.01 ;{ }^{* *}, P<0.001$ for the indicated comparisons using an unpaired Student's $t$ test. Data in $C$ and $D$ are pooled from at least three independent experiments. Data in $A, B$, and $E-I$ are representative of at least two independent experiments. 

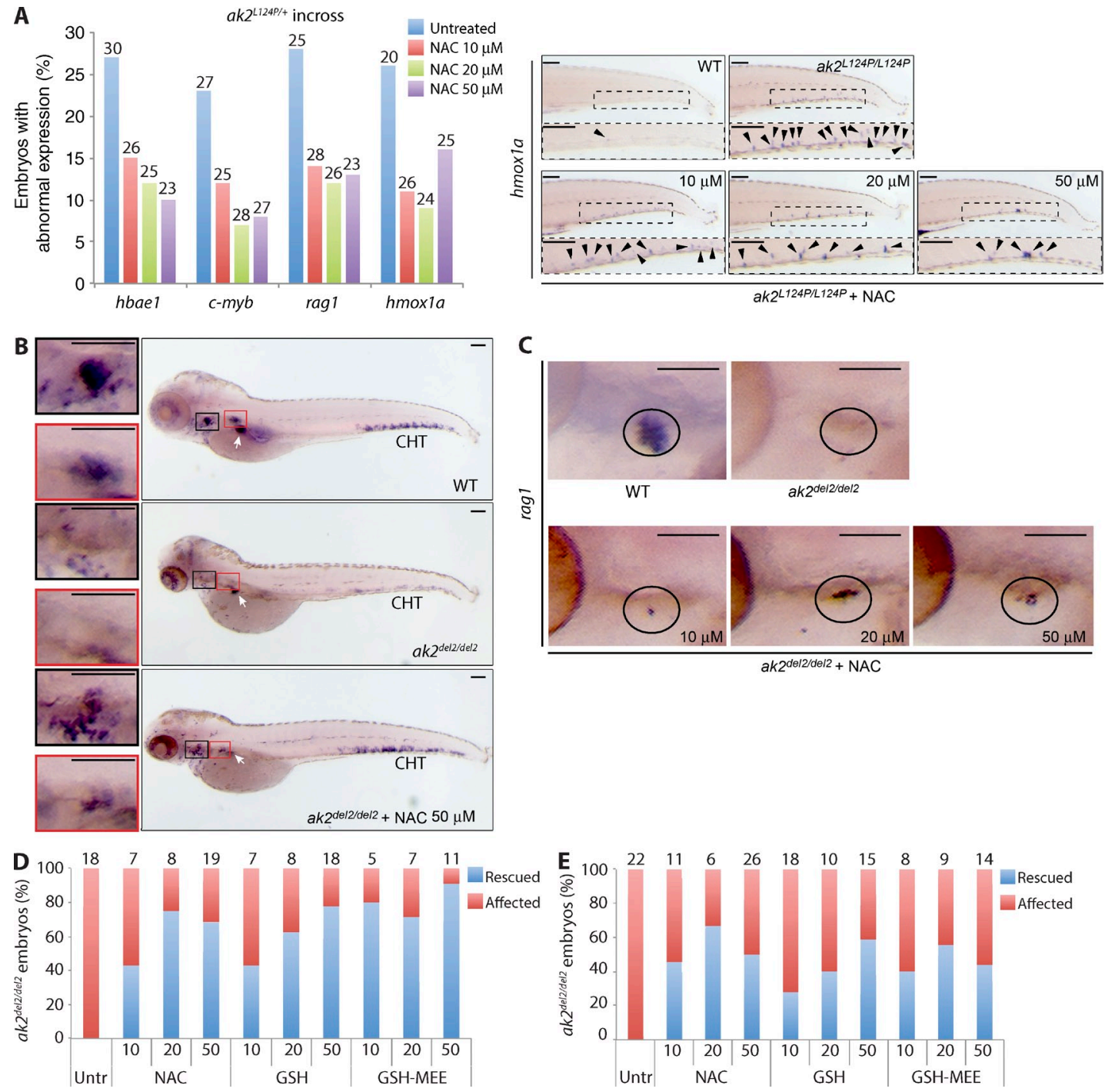

Figure 6. Antioxidant treatment induces rescue of hematopoietic phenotypes in AK2 zebrafish mutants. (A) Embryos from an incross of heterozygous ak2 ${ }^{L 124 P}$ mutants were treated with different doses of NAC $(10,20$, or $50 \mu \mathrm{M})$ until $5 \mathrm{dpf}$ when the expression of different markers was assessed by WISH. (left) Quantification of rescue induced by NAC treatment of ak2 $2^{L 124 P}$ missense mutants. Data are presented as the percentage of abnormal expression of each marker by WISH analysis at the indicated concentrations. The number of embryos analyzed is shown above each column. All observed differences compared with the untreated embryos are significant ( $\mathrm{P}<0.005$, Z-test). (right) Representative stereomicroscopy images of tail regions of WT siblings and ak2 $2^{L 24 P / L 124 P}$ mutants untreated or treated with different doses of NAC. Black arrowheads indicate $h$ mox 1a-positive cells in CHT regions (boxed insets). (B) Lateral views of WT siblings and ak2 del/2del2 mutants showing c-myb expression (WISH) in untreated and treated with $50 \mu \mathrm{M}$ NAC embryos at $5 \mathrm{dpf}$. Red and black insets show thymus and kidney regions, respectively; white arrows mark insulin expression (positive control). (C) Lateral views of rag 1 expression (WISH) in thymic region of WT siblings, ak2 del/del2 mutants untreated and treated with increasing concentrations of NAC at $5 \mathrm{dpf}$. Black circles show thymic region in each sample. (A [right], B, and C) For each sample, at least 25 embryos have been analyzed. Bars, $100 \mu \mathrm{m}$. ( $D$ and E) Quantitative analysis of the effect of antioxidant treatment on c-myb (D) and rag1 (E) expression at $5 \mathrm{dpf}$ in embryos from an incross of heterozygous ak2 del2 mutants. Data are presented as the percentage of ak2 $2^{\mathrm{de} / 2 / \mathrm{de} / 2}$ null embryos. Numbers above columns indicate the total number of ak2 ${ }^{\text {del/del2 }}$-null mutants found in each group of genotyped embryos. Data (A-E) are representative of at least two independent experiments.

\section{Antioxidant treatment rescues myeloid differentiation of AK2 ${ }^{\text {R1750/R1750 }}$ iPSCs}

Our experience in zebrafish has suggested that endogenous ROS production is increased in RD and has demonstrated efficacy of exogenous antioxidant treatment in rescuing hematopoietic developmental defects.
As a proof of concept that our in vivo findings in zebrafish can be translated into human cells, we tested the effect of antioxidant treatment in the iPSC model. GSH treatment led to a highly significant improvement in myeloid maturation in a dose-dependent manner. Maximal response was observed at a concentration of $3 \mathrm{mM}$ GSH (Fig. $9 \mathrm{~A}$ ) and led to an increase 

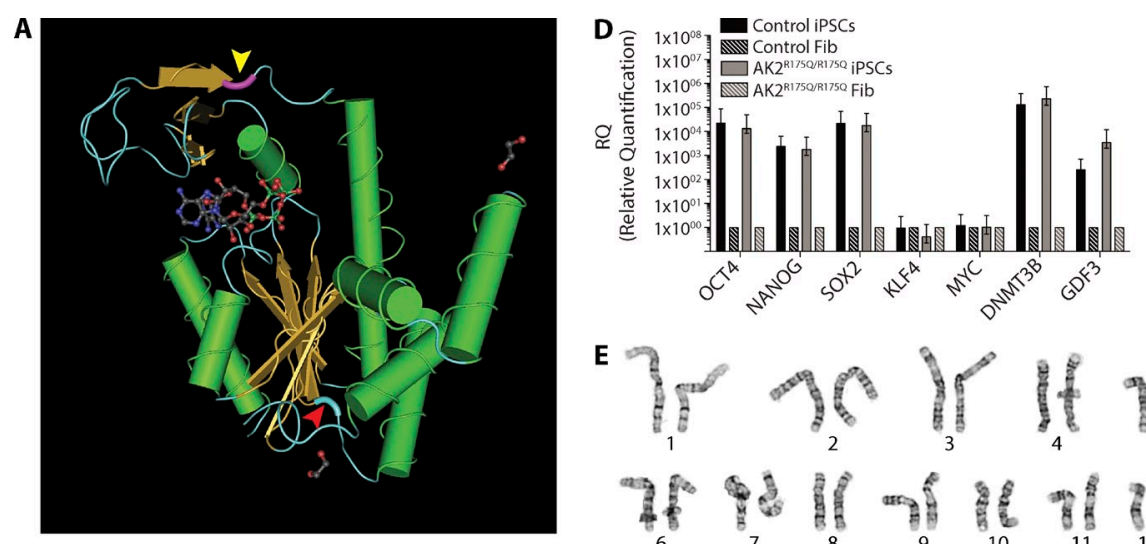

B

ZAk2 PPKEHMKDDVTGEPLIRRSDDNE hAK2 PPKEPMKDDITGEPLIRRSDDNE

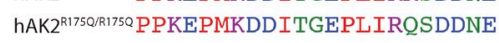
$* * * * * * * *: * * * * * * *: * * * * *$ $\Delta$

\section{C}
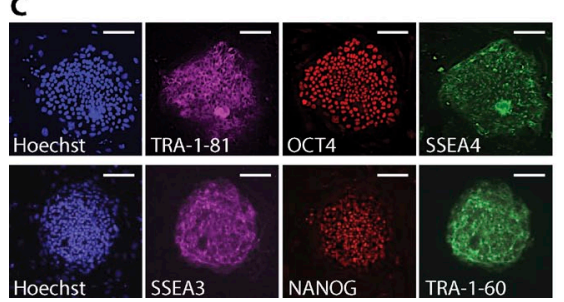

$\mathbf{E}$
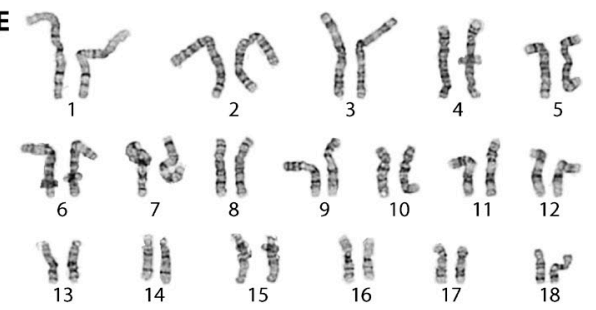

$\frac{3}{17} \quad \frac{18}{18}$

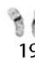

20

F ControliPSCs

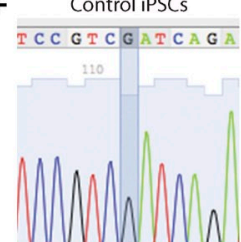

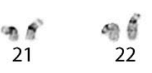
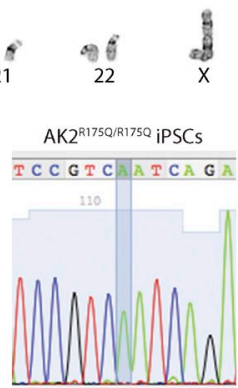

Figure 7. Generation and characterization of human AK2 ${ }^{\text {R1750/R1750 }}$ iPSCs.

(A) Schematic representation of the annotated $3 D$ structure of the human AK2 protein. The amino acid position mutated in patient-

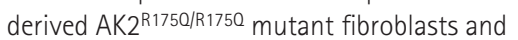
iPSC lines is highlighted in violet (yellow arrowhead). The amino acid position mutated in $a k 2^{L 124 P / L 124 P}$ mutant zebrafish is marked in light blue (red arrowhead). (B) Partial sequence multi-alignment of the LID domain of zebrafish (zAk2) and human (hAK2) proteins and the human mutated form ( $h A K 2^{\mathrm{R} 1750 / R 1750)}$. Yellow arrowhead marks the amino acid position mutated in patient-derived cell lines.

(C-F) iPSCs were generated from AK2-deficient dermal and control foreskin fibroblasts.

(C) Representative confocal microscopy images showing the expression of human pluripotency markers (TRA-1-81 and SSEA3 [magenta], Oct4 and NANOG [red], and SSEA4 and TRA-1-60 [green]) using immunofluorescently labeled antibodies in AK2 R1750/R1750 iPSCs; cellular content is highlighted by nuclear staining with Hoechst 33342 (blue). Bars, $100 \mu \mathrm{m}$. (D) mRNA analysis using quantitative real-time PCR of the indicated pluripotencyassociated genes in AK2 $2^{R 1750 / R 1750}$ and control

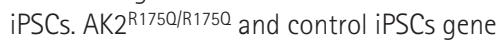
expression was compared with the respective parental fibroblasts (Fib), and human $\beta$-actin gene expression (hACTB) was used as housekeeping gene. Error bars indicate standard error. (E) Karyotype and G-banding analysis of AK2 R1750/R1750 iPSCs. (F) Sequencing of the genomic region surrounding the mutation in the control and patient-derived AK2-mutated iPSC line. Data (C-F) are representative of at least two independent experiments.

in the proportion of mature neutrophils from 2.2 to $30.7 \%$ (Fig. 9 B). In contrast, addition of compounds such as G-CSF and all-trans-retinoic acid (ATRA), which promote promyelocyte maturation in other conditions with neutropenia but have clinically proven ineffective in the treatment of RD (Castaigne et al., 1990; Bujan et al., 1993; Lagresle-Peyrou et al., 2009), had either no effect on in vitro granulocytic differentiation of AK2-mutated iPSCs (G-CSF) or even aggravated the disease phenotype (ATRA; Fig. 9 C).

\section{DISCUSSION}

$\mathrm{RD}$ is a rare and life-threatening disease affecting infants in the immediate newborn period. Although previous studies have shown that mutations in the AK2 gene are responsible for RD, the rarity of this condition and the lack of suitable animal and cellular models have represented significant obstacles to defining its molecular and cellular pathophysiology. Here we describe ak2 mutant zebrafish and patient-derived iPSCs as platforms to investigate the effects of AK2 mutations on hematopoiesis and to explore novel therapeutic approaches.

Manipulations of AK activity in different organisms results in impaired adenine nucleotide homeostasis, block of ATP export from mitochondria, and reduced cell growth and metabolism, as well as increased susceptibility to metabolic stress (Bandlow et al., 1988; Janssen et al., 2000; Pucar et al., 2000, 2002). Impairment of AK2 activity in humans leads to the severe lymphocyte and granulocyte differentiation defects and sensorineural deafness characteristic for RD (Lagresle-Peyrou et al., 2009; Pannicke et al., 2009). Our studies in zebrafish and iPSCs show that AK2 deficiency is associated with an even broader hematological phenotype than originally anticipated, which affects the development of the erythroid lineage, in addition to the known abnormalities in granulocyte and lymphocyte differentiation. Although anemia has been reported in several RD patients (Lagresle-Peyrou et al., 2009; Pannicke et al., 2009; Poliani et al., 2009), these observations have been mainly attributed to infections. Our findings in zebrafish and iPSC models strongly suggest that reduced $\mathrm{RBC}$ production may be part of the clinical phenotype of the disease.

During substrate binding, AKs undergo a conformational change in the LID and NMP domains from "open" to "closed" (Dzeja and Terzic, 2009; Daily et al., 2010). Once the catalytic process has been completed, the enzyme resumes its original configuration. This transition is important as it limits the catalytic turnover rate (Shapiro et al., 2000; Whitford et al., 2007; Daily et al., 2010). Notably, both missense mutations carried by human AK2 R175Q/R175Q iPSCs and ak2 $2^{\text {L124P/L124P }}$ zebrafish mutants are located within the catalytic region of AK2 (inside the LID domain and in a conserved region upstream of the 

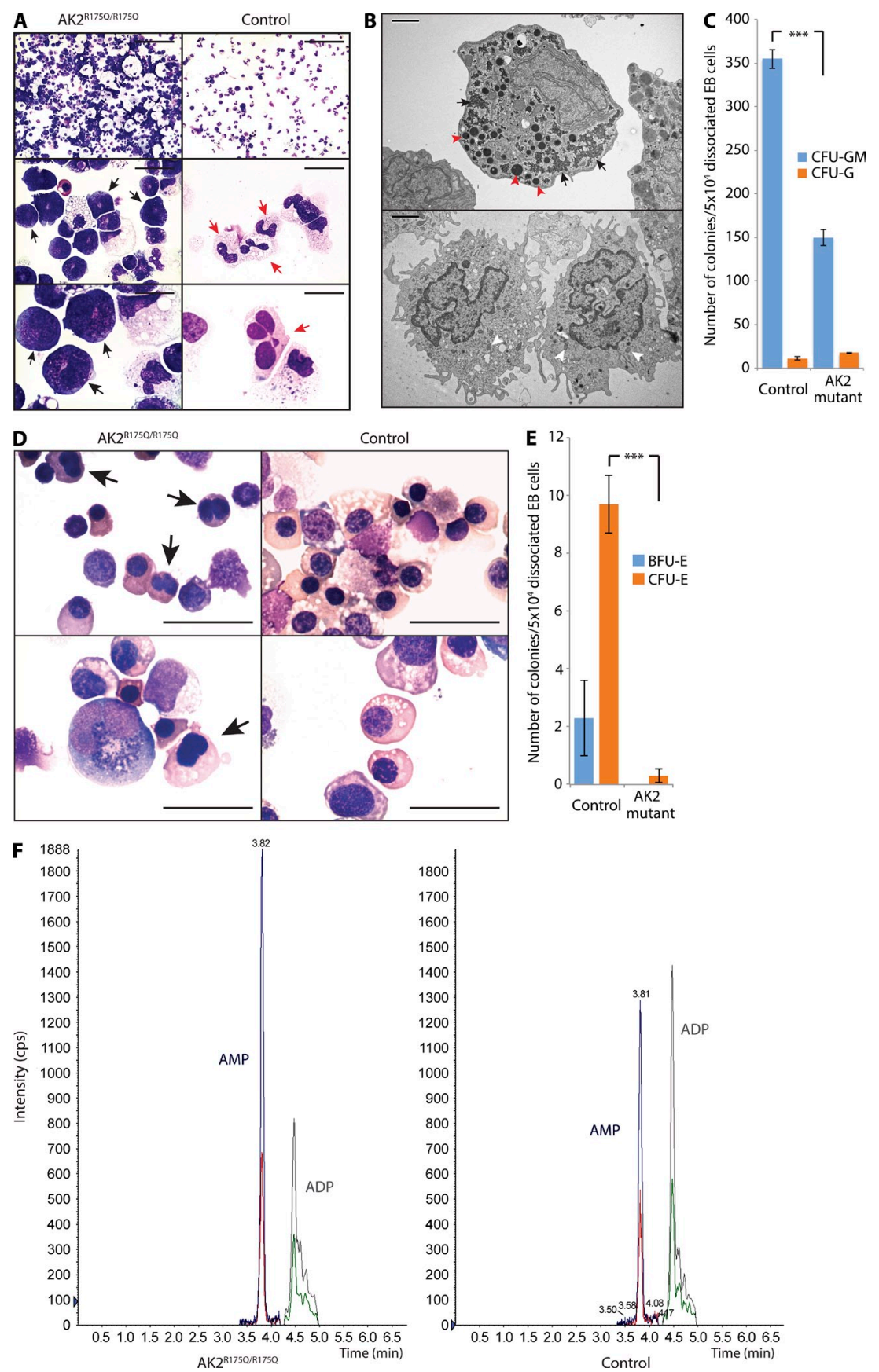

$\beta$-sheet connecting the AK2 CORE domain to the LID domain, respectively), which is expected to result in a perturbation of AK2 enzymatic activity and explains the severe phenotype observed in both models.

Our findings in zebrafish null mutants ( $a k 2^{\text {del } 2}$ and $a k 2^{\text {ins } 4}$ ) and the experiments with ak2 MOs in transgenic $\operatorname{Tg}(c d 41$ : GFP) reporter zebrafish strongly suggest that, although specification of HSPC is preserved in the absence of AK2, maturation and maintenance of this cell population in zebrafish are dependent on AK2 expression. Although further experiments are necessary to prove a direct effect of AK2 deficiency on human HSPC development, the extended phenotypic spectrum
Figure 8. AK2 deficiency affects in vitro granulopoiesis, erythropoiesis, and the adenine nucleotide profile of human myeloid cells. (A) Microscopic analysis of in vitro myeloid differentiation of

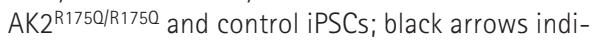
cate promyelocytes; red arrows indicate mature neutrophils. (B) Electron microscopy assessment of

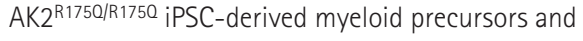
control cells. Red arrowheads mark large electrondense primary granules, and black arrows indicate glycogen storage in the cytoplasm (top). White arrowheads highlight pale secondary granules (bottom). (C) The number of myeloid colonies grown from 50k dissociated EB cells after $14 \mathrm{~d}$ of culture on methylcellulose was assessed (***, P<0.001, $\chi^{2}$ test). Data represent the mean of three experiments, and error bars depict standard error. (D) In vitro erythroid dif-

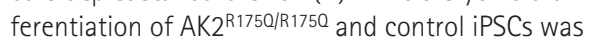
assessed by microscopy. Black arrows indicate incomplete nuclear separation. (A, $B$, and D) Data are representative of at least three independent experiments. Bars: (A, top) $100 \mu \mathrm{m}$; (A, middle) $20 \mu \mathrm{m}$; (A and $\mathrm{D}$, bottom) $10 \mu \mathrm{m}$; (B) $2 \mu \mathrm{m}$; (D, top) $25 \mu \mathrm{m}$. (E) Number of red blood cell-forming colonies (BFU-E and CFU-E) grown from 50k dissociated EB cells after $14 \mathrm{~d}$ of culture on methylcellulose $\left({ }^{* * *}, P<0.003\right.$, $\chi^{2}$ test). Data represent the mean of three independent experiments, and error bars depict standard error. (F) Quantification of cellular AMP and ADP content in AK2 ${ }^{\text {R1750/R1750 iPSC-derived myeloid cells and control }}$ myeloid cells by tandem mass spectrometry. Data are representative of three independent experiments. iPSCs generated from a human foreskin fibroblast line were used as WT control in all experiments (A-F). of myeloid and erythroid linage abnormalities observed in human iPSCs further supports this theory.

In adult bone marrow, HSPCs rely heavily on anaerobic glycolysis for energy production, keeping the generation of ROS and therefore oxidative damage to DNA to a minimum (Jang and Sharkis, 2007; Takubo et al., 2010). Although AK2 deficiency is not expected to have major bearing on glycolysis, it is expected to have a greater impact on cells relying mostly on oxidative phosphorylation to meet their metabolic needs. This could explain how the phenotypic abnormalities observed in RD become more apparent as the cell transitions from predominantly anaerobic to oxidative means of ATP 
A
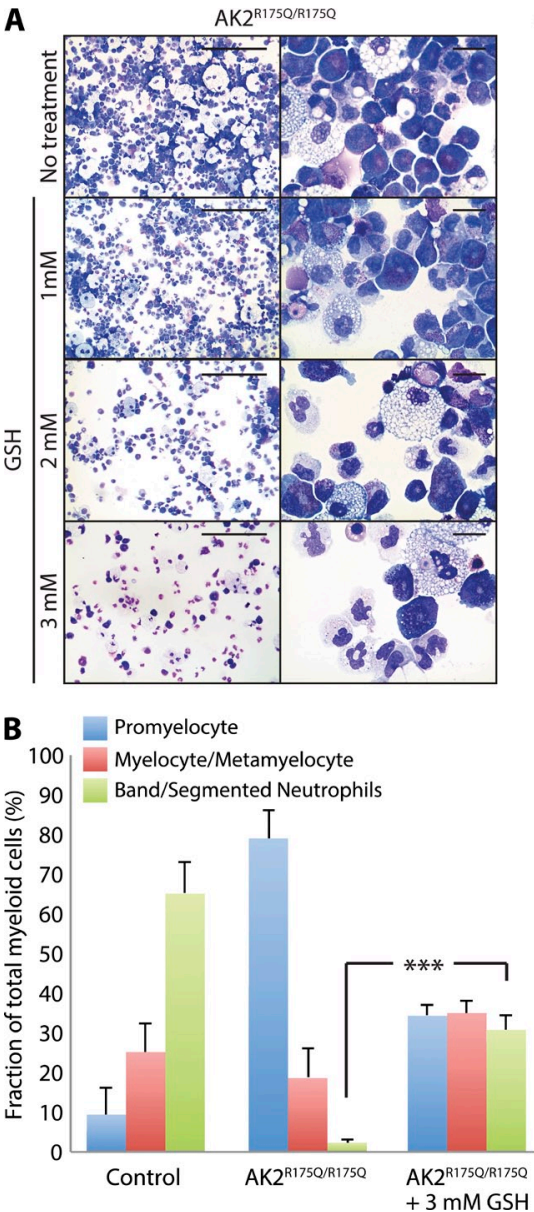

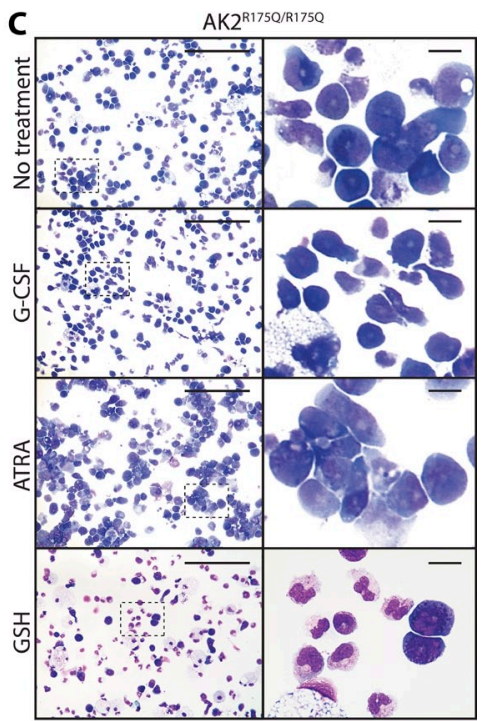

Figure 9. Antioxidant treatment rescues myeloid differentiation of human AK2 ${ }^{\text {R1750/R1750 }}$ iPSCs. (A) Micros-

copy analysis of in vitro myeloid maturation of $A K 2^{R 1750 / R 1750}$ iPSCs in the presence of different concentrations of the antioxidant agent GSH in the culture medium as indicated. Data are representative of at least three independent experiments. (B) Maturation stage-specific comparison of GSH-treated

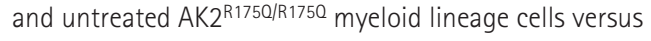
control. Data are presented as the percent fraction of total myeloid cells and represent the mean of three experiments. Error bars depict standard error (*** $P<0.0001, \chi^{2}$ test). iPSCs generated from a human foreskin fibroblast line were used as WT control. (C) Microscopy analysis of in vitro myeloid maturation of AK2 ${ }^{R 1750 / R 1750}$ iPSCs in the presence of $25 \mathrm{ng} / \mathrm{ml} \mathrm{G}-\mathrm{CSF}, 1 \mu \mathrm{M}$ ATRA, or $3 \mathrm{mM} \mathrm{GSH}$. Magnified images of boxed areas are presented in the right panels. Data are representative of at least two independent experiments. Bars: (A and $C_{1}$ left) $100 \mu \mathrm{m}$; (A and $\mathrm{C}$, right) $10 \mu \mathrm{m}$. production, which corresponds to the differentiation of HSPCs into lineage-specific mature cells. Alternatively, this might suggest a more subtle effect of AK2 deficiency on the stem cell compartment in humans that has remained unnoticed until now.

Previous studies have linked AK2 function to cell survival and proliferation. In particular, AK2 deficiency in Drosophila melanogaster is embryonically lethal (Fujisawa et al., 2009; Chen et al., 2012). Moreover, it has been suggested that AK2 deficiency may induce the unfolded protein response (Burkart et al., 2011) and apoptosis (Single et al., 1998; Köhler et al., 1999; Lee et al., 2007). The intracellular localization of AK2 is strictly limited to the mitochondrial intermembrane space (Dzeja et al., 2007) where it is required to generate ADP. This, in turn, serves as substrate for the ATP synthase and fuels the mitochondrial ATP production critical to meet the energy needs of the cell (Hatefi, 1985). Our observations in zebrafish and iPSCs point to the disturbance of cellular energy homeostasis and increased oxidative stress as key mechanisms responsible for the hematopoietic abnormalities in RD. The decrease in cellular ADP observed in AK2-mutated iPSCs is expected to have a major impact on the activity of the ATP synthase. When substrate unavailability becomes the rate-limiting factor for ATP synthesis and ATP production declines, the cell enters "state
4 respiration," leading to a transient rise in membrane potential followed by an escalation in the formation of ROS (Liesa and Shirihai, 2013). Whether this promotes mitochondrial uncoupling, resulting in a decline of the mitochondrial membrane potential (Liesa and Shirihai, 2013), or whether ROS acts as second messenger, leading to cell cycle arrest, senescence, and apoptosis (Sauer et al., 2001), remains to be investigated.

Overall, our data suggest that oxidative stress could represent a promising target for novel therapeutic approaches to this syndrome. Accordingly, our models represent a unique opportunity to study the effect of different antioxidant compounds in AK2 deficiency.

The current standard of care in RD is allogeneic HSCT (Antoine et al., 2003; Lagresle-Peyrou et al., 2009). However, the severe neutropenia and lymphopenia in this disease often lead to death before transplantation can be attempted. Furthermore, at variance with other forms of SCID, patients with RD require intensive conditioning to allow for durable donor cell engraftment (Bertrand et al., 2002), further contributing to increased morbidity and mortality. Therefore, addressing the increased oxidative stress by using antioxidant compounds may represent a previously unexploited and easyto-implement strategy to treat patients with RD and may have important implications for the development of clinical 
trials aimed at alleviating the disease phenotype, while preparing for HSCT.

\section{MATERIALS AND METHODS \\ Zebrafish lines and maintenance}

Zebrafish were maintained and used following protocols approved by the National Human Genome Research Institute Animal Care and Use Committee. Zebrafish handling, breeding, and staging were performed as previously described (Kimmel et al., 1995; Westerfield, 1995). Beginning from $24 \mathrm{hpf}$, embryos were cultured in fish water containing $0.003 \% 1$-phenyl2-thiouera (PTU) to prevent pigmentation. The following strains were used: WT EK (Ekkwill) and $\operatorname{Tg}(c d 41: G F P)$ (Lin et al., 2005) and $T g$ (fli1a: EGFP) ${ }^{r^{1}}$; $\operatorname{Tg}$ (gata1a:dsRed) ${ }^{s d 2}$ (Lawson and Weinstein, 2002; Traver et al., 2003).

\section{Databases and bioinformatic analysis of data}

The following genome assemblies were searched on the Ensembl database (Flicek et al., 2013): Homo sapiens (GRCh37.p12, Feb 2009) and Danio rerio (Zv9, Apr 2010). Multi-alignment of human and zebrafish AK2 was performed with Clustal Omega (Goujon et al., 2010). In each multi-alignment, an asterisk indicates positions that have a single, fully conserved residue, a colon indicates conservation between groups of strongly similar properties, and a period indicates conservation between groups of weakly similar properties. Structural features of amino acid sequence of human AK2 were defined based on 3D structure of human protein (MMDB ID: 37222). In all of the 3D structures, $\alpha$-helix structures are depicted in green, $\beta$-strands are depicted in yellow, and Bis(Adenosine)-5'-Tetraphosphate and 1,2-Ethanediol are shown as ball and stick models. PROVEAN score was used to predict whether a protein sequence variation affects protein function (Choi et al., 2012).

\section{Accession number}

The GenBank accession number of zebrafish ak2 isoform B is KJ651951.

\section{RT-PCR}

Total RNAs were prepared from different zebrafish embryos at different developmental stages using the RNeasy Mini kit (QIAGEN), treated with DNase I RNase-free (Roche) to avoid possible contamination from genomic DNA, and then reverse transcribed using Superscript III (Life Technologies) and random primers. The cDNAs were then subjected to PCR amplification using specific primers and Platinum Taq DNA Polymerase or Platinum Taq DNA Polymerase High Fidelity (Life Technologies) as previously described (Rissone et al., 2012). To the extent possible, all primer pairs were designed on different exons to avoid the amplification of DNA possibly contaminating cDNA preparations. Products were then separated on agarose gels at various concentrations (from 1 to $3 \%$ maximum, based on the fragments length) and visualized by ethidium bromide staining. A fragment of zebrafish $\beta$-actin cDNA was amplified by PCR ( 35 cycles) as an internal control for the quality of cDNA using a pair of primers that demonstrates the lack of genomic contamination in our RNA preparations (Argenton et al., 2004).

\section{Quantitative real-time PCR}

Quantitative real-time PCR analyses were performed in quadruplicate, and quantitative real-time PCR assays were designed using the Universal ProbeLibrary (UPL) and the ProbeFinder software (Roche). Reactions were assembled in 96-well plates (Applied Biosystems) and run under standard conditions on an ABI Prism 7900HT real-time thermocycler (Applied Biosystems). Each experiment was replicated at least three times. Specificity of the RT-PCR products was assessed by gel electrophoresis. A single product with the expected length was detected for each reaction. Data were processed using RQ Manager 1.2 and DATA assist 2.0 software and the $2^{-\Delta \Delta \mathrm{Ct}}$ method with an $\mathrm{RQ}_{\min } /$ $\mathrm{RQ}_{\max }$ confidence set at $95 \%$. The error bars indicate the calculated maximum $\left(R Q_{\max }\right)$ and minimum $\left(R_{\min }\right)$ expression levels that represent the standard errors of the mean expression level (RQ value). The upper and lower limits define the region of expression within which the true expression level value is likely to occur. See Table S1 for sequences of primers and probes used.

\section{Generation of zebrafish AK2 mutants and genotyping}

The generation of zebrafish ak2 knockout mutants using CompoZr ZFNs (Sigma-Aldrich) and the genotyping strategy have been previously described (Sood et al., 2013). We selected two mutations predicted to cause frameshifts with premature terminations, $a k 2^{\text {hg } 14}$ (c.45delAA, p.K16RfsX62) and $a k 2^{\text {hg } 15}$ (c.41insACGG, p.K16TfsX64), denoted here as $a k 2^{\text {del2 }}$ and $a k 2^{\text {ins } 4}$, respectively. We also recovered a mutant line $\left(a k 2^{\text {hg }}{ }^{16}\right.$, denoted as $\left.a k 2^{L 124 I}\right)$ carrying a c.T371C/p.L124P missense mutation within ak2 exon 4 from a zebrafish DNA library of ENU-induced mutations. DNA extraction and amplification from fixed samples were performed using Extract-N-Amp Tissue PCR kit (Sigma-Aldrich) under standard conditions and REDExtract-N-Amp PCR Ready Mix (Sigma-Aldrich). Genomic sequences flanking the point mutation of $a k 2^{L 124 P}$ mutants were PCR amplified using the following primers: AK2-F3seq (5'-TGTAAAACGACGGCCAGTTCTCATTTGTAGCTGGATGAC-3') and AK2-R4seq (5'-CAGGAAACAGCTATGACCCACTTACAGGACCCTCCATGC-3'). PCR products were sequenced with M13R primer and big-dye v3.1 sequencing mix (Life Technologies) after removal of unused primers and nucleotides with Exo-SAP-IT (Affymetrix). Sequence analysis was performed using software package Sequencher, version 5.0 (Gene Codes).

\section{WISH}

A zebrafish ak2 full-length CDS clone (clone ID 6791280; Open Biosystems, Thermo Fisher Scientific) was used as template to generate digoxigenin (DIG)-labeled antisense RNA probe for both the AK2 splicing isoforms (A and B). The following DIG-labeled antisense mRNA probes were generated using DIG RNA Labeling kit (Roche): hbae1, l-plastin, mpx, ikaros, rag1, and c-myb. For hmox $1 a$ antisense probe, gene-specific fragments were amplified by RT-PCR on suitable templates. The primers (hmox1a ISH1 FOR, 5'-TGGACTCCACCAAAAGCAAAGC-3'; and hmox1a ISH1 REV, 5'-CTTCCTCTGTGATGCTCAGCAT- $3^{\prime}$ ) were designed to cover the coding sequence of the gene of interest. PCR products were cloned into the pCR4-TOPO vector (Life Technologies), and DIG RNA Labeling kit (SP6/T7; Roche) was used to synthesize antisense or sense RNA probes. All probes were purified using Spin Post-Reaction Clean-Up Columns (Sigma-Aldrich) before use. All embryos used for WISH were fixed overnight in $4 \%$ paraformaldehyde (PFA)/PBS, rinsed with PBS-Tween, dehydrated in 100\% methanol, and stored at $-20^{\circ} \mathrm{C}$ until being processed for WISH. WISHs were performed as described by Thisse and Thisse (2008). Hybridization was performed at $70^{\circ} \mathrm{C}$. Hybridized probes were then detected by using an anti-DIG antibody conjugated to alkaline phosphatase (AP; Roche) at a 1:5,000 dilution. Nitro blue tetrazolium/5-bromo-4-chloro-3-indolyl phosphate (NBT/ BCIP; Roche) or INT/BCIP Stock Solution (Roche) was used as a substrate for AP. Stained embryos were stored in 4\% PFA until imaging.

\section{Sudan black and acridine orange stainings}

For Sudan black staining, embryos were fixed with 4\% PFA (Polysciences) in PBS for $2 \mathrm{~h}$ at room temperature, rinsed in PBS, and incubated in Sudan black solution (Sigma-Aldrich) as described previously (Sheehan and Storey, 1947; Le Guyader et al., 2008). For acridine orange staining, zebrafish larvae were anesthetized with Tricaine (Sigma-Aldrich), incubated in a solution of $3 \mu \mathrm{g} / \mathrm{ml}$ acridine orange (Sigma-Aldrich) in E3 medium with Tricaine (Sigma-Aldrich) for $30 \mathrm{~min}$, washed twice in E3 with Tricaine, and then analyzed under a fluorescent stereomicroscope.

\section{TUNEL assay}

TUNEL assay was performed as previously described (Finckbeiner et al., 2011) with minor modifications. After incubation in STOP/Wash solution followed by $3 \times 10 \mathrm{~min}$ PBT washes, the embryos were incubated in anti-DIG Fluorescein-conjugated antibody (EMD Millipore) or in anti-DIG APconjugated antibody (Roche) using NBT/BCIP (Roche) as substrates for AP.

\section{Cell sorting and oxidative stress staining}

Whole mount staining with the MitoSOX and CellROX oxidative stress probes was performed as previously described (Mugoni et al., 2014) on living 
zebrafish embryos at different stages of development. Embryos from incross of $a k 2^{\text {ins } 4 /+}$ and $a k 2^{L 124 P /+}$ were subjected to tail resection $30 \mathrm{~min}$ before being stained with $5 \mu \mathrm{M}$ MitoSOX or CellROX solutions for $30 \mathrm{~min}$, followed by fluorescence analysis using confocal microscopy. At the end of the analysis, embryos were recovered and genotyped. Quantitative analysis of the oxidative stress using flow cytometry was performed as previously described (Mugoni et al., 2014) with the following modifications. Embryos from incross of $a k 2^{\text {del2 } /+}$ homozygous fish were grown until the selected stage ( 4 or $5 \mathrm{dpf}$ ), and then null mutants and their siblings ( $\sim 60$ embryos each) were separated and collected in different tubes. After euthanasia, embryos were washed twice with $1 \times$ PBS and then dissociated with a mix of collagenase P and Trypsin-EDTA in $1 \times$ PBS at $28^{\circ} \mathrm{C}$ for $20 \mathrm{~min}$. Homogenization of the samples was obtained by extensive periodical pipetting. Then the cells were maintained in DMEM 10\% FCS at $28^{\circ} \mathrm{C}$ until staining. For MitoSOX analysis, cells were resuspended in HBSS containing calcium and magnesium and then stained with 5-2.5 $\mu \mathrm{M}$ MitoSOX Red (Life Technologies) for 15 min. For CellROX Green (Life Technologies) staining, the probe was added to the DMEM 10\% FCS medium at a final concentration of 2.5-1 $\mu \mathrm{M}$ for $30 \mathrm{~min}$. For Annexin V-APC and 7-AAD (BD) double staining, cells were washed and resuspended in $1 \times$ binding buffer and then incubated with the probes according to the manufacturer's instruction. In all cases, the cells were incubated at $28^{\circ} \mathrm{C}$ in the dark and then washed twice to remove excess probe. Flow cytometry data were collected on a FACSAria II equipped with FACSDiva software using the blue 488-nm and red 640-nm lasers (BD). Further data analysis was performed using FlowJo software (FlowJo, LLC). Unstained cells were used to set the gates in the FlowJo program.

\section{Imaging}

The embryos obtained by WISH, Sudan black staining, acridine orange, and TUNEL assay were observed with an MZ16F stereomicroscope (Leica), and pictures were taken with a DC500 camera using a FireCam (version 1.7.1, Leica). Confocal images were acquired at room temperature using an LSM 510 NLO Meta system mounted on an Axiovert 200M microscope (Carl Zeiss) with a Plan-Apochromat $10 \times / 0.45$ or a Plan-Apochromat $20 \times / 0.75$ objective lens. Excitation wavelengths of 488, 561, and $770 \mathrm{~nm}$ were used for detection of EGFP, dsRed, and DAPI, respectively. A range of z-slices was used depending on the zebrafish orientation to capture all desired structures using optical slices of $\sim 4 \mu \mathrm{m}$. All confocal images were of frame size 512 pixels by 512 pixels, scan zoom of $0.7 \times$, and line averaged four times. Images were collected, and maximum projections were processed using the ZEN 2009V5.5 SP2 software package (Carl Zeiss).

\section{Morpholino injection}

Antisense morpholinos targeting the $a k 2$ ATG region ( $a k 2 \mathrm{MO} 1,5^{\prime}$-CATGGCTACAGCTTCTTTACTAACT-3') or the splice acceptor site of $a k 2$ intron 3 (ak2 MO2; Pannicke et al., 2009) and the standard control MO (ST-CTRL MO, 5'-CCTCTTACCTCAGTTACAATTTATA-3') were manufactured by Gene Tools. The morpholinos were used according to the manufacturer's instructions, and the indicated amount of each morpholino was injected into the yolk of one-cell-stage WT embryos. To confirm that AK2 pre-mRNA was specifically targeted, RT-PCR was performed on ak2 MO2-injected embryos. RT-PCR on ST-CTRL MO-injected embryos was included as a control.

\section{Drug exposure}

All compounds used in these experiments were purchased from Sigma-Aldrich. Zebrafish embryos were exposed to different doses of NAC, L-Glutathione reduced (GSH), or GSH-MEE from $\sim 10$ hpf until $5 \mathrm{dpf}$ in embryo medium containing $0.003 \%$ PTU. New embryo medium with fresh compound was administered daily until $5 \mathrm{dpf}$. After exposure, fish were fixed and the expression of different markers was tested by WISH. Embryos were phenotyped based on the expression of the markers and then genotyped to verify the classification.

\section{Cell lines and culture}

Reprogramming. Human dermal fibroblasts were collected from a patient with homozygous c.524G $>$ A mutation in the human $A K 2$ gene (Henderson et al., 2013) by punch biopsy. All human protocols were approved by Boston Children's Hospital Institutional Review Board. Fibroblasts were expanded in hFIB medium containing DMEM, 20\% FBS, 1 mmol/liter L-Glutamine, $100 \mathrm{mmol} /$ liter nonessential amino acids, and 1\% PenStrep. Fibroblasts were reprogrammed using an excisable, self-inactivating, codon-optimized lentiviral vector carrying OCT4, SOX2, KLF4, cMYC, and dTomato cDNAs as previously described (Warlich et al., 2011). Transduction was performed with a starting cell count of $3 \times 10^{4}$ fibroblasts using a multiplicity of infection (MOI) of 1. iPSC colonies emerged after 17-21 d of culture. To generate control iPSCs, human neonatal foreskin fibroblast "BJ" (ATCC CRL-2522) was used, and reprogramming was performed as described above.

iPSC culture. iPSCs were maintained on irradiated mouse embryonic fibroblasts (Global Stem) in hESC medium containing DMEM, 20\% KOSR, $10 \mathrm{ng} / \mathrm{ml} \mathrm{bFGF}, 1 \mathrm{mmol} /$ liter L-Glutamine, $100 \mathrm{mmol} /$ liter nonessential amino acids, $100 \mathrm{mmol} /$ liter 2-b-Mercaptoethanol, and $100 \mathrm{mg} /$ liter Primocin as previously described (Park et al., 2008b). Karyotype analysis was performed by Cell Line Genetics. Genomic DNA was isolated using the QiAMP DNA kit (QIAGEN), and the genomic regions flanking the reported mutations were PCR amplified using primers AK2 M13 F (5'-ACAGCATCCTGGGCAGAATG-3') and AK2 M13 R (5'-ATTCCCACCCATTGCCCTAC-3') and sequenced in a commercial core facility (Eton Bioscience).

Immunohistochemistry. iPSCs were selected based on morphology, subcloned for five to seven passages, and then manually picked as single-colony cells and seeded into a 96-well plate wells containing $8.5 \times 10^{3}$ irradiated mouse embryonic fibroblasts per well in standard hESC medium. Cells were washed with PBS, fixed at room temperature in 4\% PFA/PBS for $15 \mathrm{~min}$, washed with PBS, and permeabilized/blocked for $1 \mathrm{~h}$ at room temperature using $0.1 \%$ Triton X-100 in animal-free blocker (Vector Laboratories) followed by three PBS washes. Cells were subsequently incubated with primary antibodies (OCT4: clone 40/oct-3, Alexa Fluor 555, 1:50 dilution; NANOG: N31-355, PE, 1:30; SSEA4: MC813-70, Alexa Fluor 488, 1:200; SSEA3: MC-631, Alexa Fluor 647, 1:30;TRA-1-81: Alexa Fluor 647, 1:30;TRA-160: Alexa Fluor 488, 1:50; all from BD) and $2 \mu \mathrm{g} / \mathrm{ml} \mathrm{Hoechst} 33342$ in animal-free blocker at $4^{\circ} \mathrm{C}$ overnight, washed, and imaged on a Pathway 435 bioimager $(\mathrm{BD})$ using a $10 \times$ lens. Background images (empty well images) were subtracted, and images were cropped, pseudocolored, and assembled into figure panels using the ImageJ software (National Institutes of Health). Immunofluorescence intensity was compared with a control hES cell line as internal standard to yield semiquantitative results.

Quantitative real-time PCR. RNA was extracted from $10^{6}$ cells using the mirVana RNA isolation kit (Ambion) and reverse transcribed to cDNA with qScript cDNA supermix (Quanta Bioscience) according to the manufacturer's instructions. Expression of the OCT4, KLF4, $C M Y C, S O X 2$, NANOG, GDF3, and DNMT3B genes was quantified by real-time PCR with Power SYBR Green PCR Master Mix (Applied Biosystems) on a 7500 Real Time PCR analyzer (Applied Biosystems) and normalized to the human $\beta$-actin gene expression ( $h A C T B)$ using primers previously reported (Park et al., 2008b). Relative expression compared with parental fibroblasts was calculated using the $\Delta \Delta \mathrm{Ct}$ (ddCt) method.

\section{Directed myeloid differentiation}

EBs were generated in suspension culture from iPSCs using a bFGF-free hES cell medium containing $300 \mathrm{ng} / \mathrm{ml} \mathrm{SCF}, 300 \mathrm{ng} / \mathrm{ml} \mathrm{FLT3,} 50 \mathrm{ng} / \mathrm{ml} \mathrm{G-CSF}$, $10 \mathrm{ng} / \mathrm{ml} \mathrm{IL3,10} \mathrm{ng/ml} \mathrm{IL6,} \mathrm{and} 50 \mathrm{ng} / \mathrm{ml} \mathrm{BMP} 4$ on low-adhesion plates as described previously (Park et al., 2008a). After 14 d, EBs were dissociated using $0.5 \mathrm{mg} / \mathrm{ml}$ Collagenase B, nonenzymatic cell dissociation buffer (Gibco) and mechanical sheering. EB-derived single cells were plated on methylcellulose (MethoCult; STEMCELL Technologies) and cultured for 14 further days according to the manufacturer's instructions in $21 \% \mathrm{O}_{2}, 5 \% \mathrm{CO}_{2}$. For samples grown in the presence of GSH, a $100 \mathrm{mM}$ stock solution was freshly prepared from L-Glutathione (reduced; Sigma-Aldrich) and added to the methylcellulose to yield a final concentration of $1-3 \mathrm{mM}$. For samples grown 
in the presence of ATRA and G-CSF, retinoic acid powder (Sigma-Aldrich) was reconstituted in DMSO to make a $1 \mathrm{mM}$ stock solution and added to methylcellulose to a final concentration of $1 \mu \mathrm{M}$. G-CSF (Neupogen) powder was reconstituted in sterile PBS with $0.1 \%$ serum albumin and added to methylcellulose to a final concentration of $25 \mathrm{ng} / \mathrm{ml}$.

\section{Electron microscopy}

One million iPSC-derived myeloid cells were pelleted and resuspended in $200 \mu \mathrm{l}$ of $25 \%$ glutaraldehyde and $200 \mu \mathrm{l}$ PFA/picric acid. Sections were cut and grits prepared by the Harvard Medical School Electron Microscopy Core Facility. Images were obtained using a Tecnai $\mathrm{G}^{2}$ Spirit BioTWIN microscope (FEI).

\section{Tandem mass spectrometry}

Shock-frozen pellets from 1-2 $\times 10^{6}$ iPSC-derived myeloid cells were reconstituted in $500 \mu \mathrm{l}$ water and sonicated on ice for $10 \mathrm{~s}$. Lysate volumes of equal total cell numbers were precipitated by adding $250-\mu \mathrm{l}$ volume of acetonitrile and centrifuged at 16,200 rpm for $2 \mathrm{~min}$. Supernatant was transferred to a new vial, dried under nitrogen flow, and resuspended in $50 \mu \mathrm{l}$ water. A calibration curve, containing a mixture of ATP, ADP, and AMP, ranging from 0 to $10 \mu \mathrm{mol} /$ liter $(0,0.01,0.1,1$, and $10 \mu \mathrm{mol} / \mathrm{liter})$ was used to quantify nucleotides. Nucleotide quantification was performed using a Triple-Quad Mass Spectrometer (8040; Shimadzu) equipped with a turbo ion spray source and coupled to a Nexera UPLC (Shimadzu).

\section{Statistical analysis}

Statistical analyses were performed using Excel software (Microsoft). Asterisks refer to $\mathrm{p}$-values. P-values and statistical tests applied are listed in the figure legends.

\section{Online supplemental material}

Table S1 shows sequences of primers and quantitative PCR probes used in this study. Online supplemental material is available at http://www.jem.org/ cgi/content/full/jem.20141286/DC1.

We thank Drs. Paul Liu and Erica Bresciani for helpful discussions and advice and Drs. Milton English and Blake Trevor for early efforts in the project. We thank Wendy London and Guo Dongjing for the statistical analysis on all iPSC experiments.

This work was supported in part by grant 5R01Al100887 from the National Institute of Allergy and Infectious Diseases (NIAID) and by the Manton Foundation (to L.D. Notarangelo), by funding from the Intramural Research Program of the National Human Genome Research Institute (to F. Candotti, R. Sood, and S. Chandrasekharappa), by UNIL-CHUV grant CGRB 29583 (to F. Candotti), by EU FP7 program Cellpid and Cluster of Excellence REBIRTH (DFG; to A. Schambach), and by the Kuwait Foundation for Advancement of Science (2010-1302-05; to W. Al-Herz). K.G. Weinacht's work was supported by the Amy Potter Fellowship, the Charles King Trust, and the Primary Immune Deficiency Treatment Consortium and funded by grant 1U54Al082973 from NIAID.

The authors declare no competing financial interests.

Author contributions: A. Rissone and F. Candotti designed, conducted, and analyzed the zebrafish experiments. K.G. Weinacht and L.D. Notarangelo designed, conducted, and analyzed the iPSC experiments. A. Rissone, K.G. Weinacht, L.D. Notarangelo, and F. Candotti wrote the manuscript; L.D. Notarangelo and F. Candotti supervised the project. G. la Marca and E. Giocaliere performed the tandem mass spectrometry. A. Rissone, K. Bishop, and R. Sood generated the zebrafish mutants. A. Rissone, K. Bishop, S. Chandrasekharappa, and M. Jones performed the genotyping of zebrafish mutants. J. Jagadeesh and K.L. Simon provided intellectual input. A. Rissone and M. Kirby performed the flow cytometer analysis on zebrafish samples. A. Rissone and S. Wincovitch performed confocal analysis on zebrafish embryos. W. Al-Herz provided the patient's skin biopsy. A. Schambach provided the reprogramming lentiviral vector for iPSC generation. A. DeVine, T. Schlaeger, and Y. Itan participated in the characterization of iPSCs. K. Felgentreff and K. Dobbs helped with culture of iPSCs. All authors read and approved the manuscript.

Submitted: 7 July 2014

Accepted: 1 June 2015

\section{REFERENCES}

Al-Zahrani, D., A. Al-Ghonaium, H. Al-Mousa, A. Al-Kassar, and C.M. Roifman. 2013. Skeletal abnormalities and successful hematopoietic stem cell transplantation in patients with reticular dysgenesis. J. Allergy Clin. Immunol. 132:993-996. http://dx.doi.org/10.1016/j.jaci.2013.04.055

Aloj, G., G. Giardino, L. Valentino, F. Maio, V. Gallo, T. Esposito, R. Naddei, E. Cirillo, and C. Pignata. 2012. Severe combined immunodeficiences: new and old scenarios. Int. Rev. Immunol. 31:43-65. http:// dx.doi.org/10.3109/08830185.2011.644607

Antoine, C., S. Müller, A. Cant, M. Cavazzana-Calvo, P. Veys, J. Vossen, A. Fasth, C. Heilmann, N. Wulffraat, R. Seger, et al. European Society for Immunodeficiency. 2003. Long-term survival and transplantation of haemopoietic stem cells for immunodeficiencies: report of the European experience 1968-99. Lancet. 361:553-560. http://dx.doi.org/10.1016/ S0140-6736(03)12513-5

Argenton, F., S. Giudici, G. Deflorian, S. Cimbro, F. Cotelli, and M. Beltrame. 2004. Ectopic expression and knockdown of a zebrafish sox21 reveal its role as a transcriptional repressor in early development. Mech. Dev. 121:131-142. http://dx.doi.org/10.1016/j.mod.2004.01.001

Bandlow, W., G. Strobel, C. Zoglowek, U. Oechsner, and V. Magdolen. 1988. Yeast adenylate kinase is active simultaneously in mitochondria and cytoplasm and is required for non-fermentative growth. Eur. J. Biochem. 178:451-457. http://dx.doi.org/10.1111/j.1432-1033.1988 .tb14469.x

Bertrand, J.Y., A.D. Kim, S. Teng, and D. Traver. 2008. CD $41^{+} \mathrm{cmyb}^{+}$ precursors colonize the zebrafish pronephros by a novel migration route to initiate adult hematopoiesis. Development. 135:1853-1862. http:// dx.doi.org/10.1242/dev.015297

Bertrand, Y., S.M. Müller, J.L. Casanova, G. Morgan, A. Fischer, and W. Friedrich. 2002. Reticular dysgenesis: HLA non-identical bone marrow transplants in a series of 10 patients. Bone Marrow Transplant. 29:759762. http://dx.doi.org/10.1038/sj.bmt.1703531

Bujan, W., A. Ferster, E. Sariban, and W. Friedrich. 1993. Effect of recombinant human granulocyte colony-stimulating factor in reticular dysgenesis. Blood. 82:1684.

Burkart, A., X. Shi, M. Chouinard, and S. Corvera. 2011. Adenylate kinase 2 links mitochondrial energy metabolism to the induction of the unfolded protein response. J. Biol. Chem. 286:4081-4089. http://dx.doi .org/10.1074/jbc.M110.134106

Castaigne, S., C. Chomienne, M.T. Daniel, P. Ballerini, R. Berger, P. Fenaux, and L. Degos. 1990. All-trans retinoic acid as a differentiation therapy for acute promyelocytic leukemia. I. Clinical results. Blood. 76:1704-1709.

Chen, R.P., C.Y. Liu, H.L. Shao, W.W. Zheng, J.X. Wang, and X.F. Zhao. 2012. Adenylate kinase 2 (AK2) promotes cell proliferation in insect development. BMC Mol. Biol. 13:31. http://dx.doi.org/10.1186/ 1471-2199-13-31

Choi, Y., G.E. Sims, S. Murphy, J.R. Miller, and A.P. Chan. 2012. Predicting the functional effect of amino acid substitutions and indels. PLoS ONE. 7:e46688. http://dx.doi.org/10.1371/journal.pone.0046688

Craven, S.E., D. French, W. Ye, F. de Sauvage, and A. Rosenthal. 2005. Loss of $\mathrm{Hspa} 9 \mathrm{~b}$ in zebrafish recapitulates the ineffective hematopoiesis of the myelodysplastic syndrome. Blood. 105:3528-3534. http://dx.doi .org/10.1182/blood-2004-03-1089

Daily, M.D., G.N. Phillips Jr., and Q. Cui. 2010. Many local motions cooperate to produce the adenylate kinase conformational transition. J. Mol. Biol. 400:618-631. http://dx.doi.org/10.1016/j.jmb.2010.05.015

De Vaal, O.M., and V. Seynhaeve. 1959. Reticular dysgenesia. Lancet. 274:1123-1125. http://dx.doi.org/10.1016/S0140-6736(59)90105-9

Dzeja, P.P., and A. Terzic. 2003. Phosphotransfer networks and cellular energetics. J. Exp. Biol. 206:2039-2047. http://dx.doi.org/10.1242/ jeb.00426

Dzeja, P., and A. Terzic. 2009. Adenylate kinase and AMP signaling networks: metabolic monitoring, signal communication and body energy sensing. Int. J. Mol. Sci. 10:1729-1772. http://dx.doi.org/10.3390/ijms10041729

Dzeja, P.P., C. Shung, and A. Terzic. 2007. Integration of adenylate kinase and glycolytic and clycogenolytic circuits in cellular energetics. In Molecular System Bioenergetics: Energy for Life.V. Saks, editor. Wiley-VCH Verlag GmbH \& Co. KGaA, Weinheim, Germany. 265-301. 
Finckbeiner, S., P.J. Ko, B. Carrington, R. Sood, K. Gross, B. Dolnick, J. Sufrin, and P. Liu. 2011. Transient knockdown and overexpression reveal a developmental role for the zebrafish enosf1b gene. Cell Biosci. 1:32. http://dx.doi.org/10.1186/2045-3701-1-32

Fischer, A., F. Le Deist, S. Hacein-Bey-Abina, I. André-Schmutz, G.S. Basile, J.P. de Villartay, and M. Cavazzana-Calvo. 2005. Severe combined immunodeficiency. A model disease for molecular immunology and therapy. Immunol. Rev. 203:98-109. http://dx.doi.org/10.1111/ j.0105-2896.2005.00223.x

Flicek, P., I. Ahmed, M.R. Amode, D. Barrell, K. Beal, S. Brent, D. CarvalhoSilva, P. Clapham, G. Coates, S. Fairley, et al. 2013. Ensembl 2013. Nucleic Acids Res. 41:D48-D55. http://dx.doi.org/10.1093/nar/gks1236

Fujisawa, K., R. Murakami, T. Horiguchi, and T. Noma. 2009. Adenylate kinase isozyme 2 is essential for growth and development of Drosophila melanogaster. Comp. Biochem. Physiol. B Biochem. Mol. Biol. 153:29-38. http://dx.doi.org/10.1016/j.cbpb.2009.01.006

Goujon, M., H. McWilliam, W. Li, F. Valentin, S. Squizzato, J. Paern, and R. Lopez. 2010. A new bioinformatics analysis tools framework at EMBL-EBI. Nucleic Acids Res. 38:W695-W699. http://dx.doi.org/ $10.1093 / \mathrm{nar} / \mathrm{gkq} 313$

Hatefi, Y. 1985. The mitochondrial electron transport and oxidative phosphorylation system. Annu. Rev. Biochem. 54:1015-1069. http://dx.doi .org/10.1146/annurev.bi.54.070185.005055

Henderson, L.A., F. Frugoni, G. Hopkins, W. Al-Herz, K. Weinacht, A.M. Comeau, F.A. Bonilla, L.D. Notarangelo, and S.Y. Pai. 2013 First reported case of Omenn syndrome in a patient with reticular dysgenesis. J. Allergy Clin. Immunol. 131:1227-1230: e1-e3. http://dx.doi .org/10.1016/j.jaci.2012.07.045

Jang, Y.Y., and S.J. Sharkis. 2007. A low level of reactive oxygen species selects for primitive hematopoietic stem cells that may reside in the lowoxygenic niche. Blood. 110:3056-3063. http://dx.doi.org/10.1182/ blood-2007-05-087759

Janssen, E., P.P. Dzeja, F. Oerlemans, A.W. Simonetti, A. Heerschap, A. de Haan, P.S. Rush, R.R. Terjung, B. Wieringa, and A. Terzic. 2000. Adenylate kinase 1 gene deletion disrupts muscle energetic economy despite metabolic rearrangement. EMBO J. 19:6371-6381. http://dx.doi .org/10.1093/emboj/19.23.6371

Kimmel, C.B., W.W. Ballard, S.R. Kimmel, B. Ullmann, and T.F. Schilling. 1995. Stages of embryonic development of the zebrafish. Dev. Dyn. 203:253-310. http://dx.doi.org/10.1002/aja.1002030302

Kissa, K., E. Murayama, A. Zapata, A. Cortés, E. Perret, C. Machu, and P. Herbomel. 2008. Live imaging of emerging hematopoietic stem cells and early thymus colonization. Blood. 111:1147-1156. http://dx.doi.org/ 10.1182/blood-2007-07-099499

Köhler, C., A. Gahm, T. Noma, A. Nakazawa, S. Orrenius, and B. Zhivotovsky. 1999. Release of adenylate kinase 2 from the mitochondrial intermembrane space during apoptosis. FEBS Lett. 447:10-12.

Lagresle-Peyrou, C., E.M. Six, C. Picard, F. Rieux-Laucat, V. Michel, A. Ditadi, C. Demerens-de Chappedelaine, E. Morillon, F. Valensi, K.L. Simon-Stoos, et al. 2009. Human adenylate kinase 2 deficiency causes a profound hematopoietic defect associated with sensorineural deafness. Nat. Genet. 41:106-111. http://dx.doi.org/10.1038/ng.278

Lagresle-Peyrou, C., B. Neven, E. Six, C. Picard, C. Demerens-de Chappedelaine, Y. Bertrand, N. Jabado, C. Chomienne, I. RadfordWeiss, C. Brouzes, et al. 2011. Occurrence of myelodysplastic syndrome in 2 patients with reticular dysgenesis. J. Allergy Clin. Immunol. 128:230232: e2. http://dx.doi.org/10.1016/j.jaci.2011.02.008

Lawson, N.D., and B.M. Weinstein. 2002. In vivo imaging of embryonic vascular development using transgenic zebrafish. Dev. Biol. 248:307318. http://dx.doi.org/10.1006/dbio.2002.0711

Le Guyader, D., M.J. Redd, E. Colucci-Guyon, E. Murayama, K. Kissa, V. Briolat, E. Mordelet, A. Zapata, H. Shinomiya, and P. Herbomel. 2008. Origins and unconventional behavior of neutrophils in developing zebrafish. Blood.111:132-141.http://dx.doi.org/10.1182/blood-2007-06095398

Lee, H.J., J.O. Pyo, Y. Oh, H.J. Kim, S.H. Hong, Y.J. Jeon, H. Kim, D.H. Cho, H.N. Woo, S. Song, et al. 2007. AK2 activates a novel apoptotic pathway through formation of a complex with FADD and caspase-10. Nat. Cell Biol. 9:1303-1310. http://dx.doi.org/10.1038/ncb1650
Lee, Y., J.W. Kim, S.M. Lee, H.J. Kim, K.S. Lee, C. Park, and I.S. Choe. 1998. Cloning and expression of human adenylate kinase 2 isozymes: differential expression of adenylate kinase 1 and 2 in human muscle tissues. J. Biochem. 123:47-54. http://dx.doi.org/10.1093/oxfordjournals .jbchem.a021915

Liesa, M., and O.S. Shirihai. 2013. Mitochondrial dynamics in the regulation of nutrient utilization and energy expenditure. Cell Metab. 17:491-506. http://dx.doi.org/10.1016/j.cmet.2013.03.002

Lin, H.F., D. Traver, H. Zhu, K. Dooley, B.H. Paw, L.I. Zon, and R.I. Handin. 2005. Analysis of thrombocyte development in CD41-GFP transgenic zebrafish. Blood. 106:3803-3810. http://dx.doi.org/10.1182/blood2005-01-0179

Liu, R., A.L. Ström, J. Zhai, J. Gal, S. Bao, W. Gong, and H. Zhu. 2009. Enzymatically inactive adenylate kinase 4 interacts with mitochondrial ADP/ATP translocase. Int. J. Biochem. Cell Biol. 41:1371-1380. http:// dx.doi.org/10.1016/j.biocel.2008.12.002

Mugoni, V., A. Camporeale, and M.M. Santoro. 2014. Analysis of oxidative stress in zebrafish embryos. J. Vis. Exp. (89):e51328. http://dx.doi .org/10.3791/51328

Pannicke, U., M. Hönig, I. Hess, C. Friesen, K. Holzmann, E.M. Rump, T.F. Barth, M.T. Rojewski, A. Schulz, T. Boehm, et al. 2009. Reticular dysgenesis (aleukocytosis) is caused by mutations in the gene encoding mitochondrial adenylate kinase 2. Nat. Genet. 41:101-105. http://dx.doi .org $/ 10.1038 / \mathrm{ng} .265$

Park, I.H., N. Arora, H. Huo, N. Maherali, T. Ahfeldt, A. Shimamura, M.W. Lensch, C. Cowan, K. Hochedlinger, and G.Q. Daley. 2008a. Disease-specific induced pluripotent stem cells. Cell. 134:877-886. http:// dx.doi.org/10.1016/j.cell.2008.07.041

Park, I.H., R. Zhao, J.A. West, A. Yabuuchi, H. Huo, T.A. Ince, P.H. Lerou, M.W. Lensch, and G.Q. Daley. 2008b. Reprogramming of human somatic cells to pluripotency with defined factors. Nature. 451:141-146. http://dx.doi.org/10.1038/nature06534

Poliani, P.L., F. Facchetti, M. Ravanini, A.R. Gennery, A. Villa, C.M. Roifman, and L.D. Notarangelo. 2009. Early defects in human T-cell development severely affect distribution and maturation of thymic stromal cells: possible implications for the pathophysiology of Omenn syndrome. Blood. 114:105-108. http://dx.doi.org/10.1182/blood-2009-03-211029

Pucar, D., E. Janssen, P.P. Dzeja, N. Juranic, S. Macura, B. Wieringa, and A. Terzic. 2000. Compromised energetics in the adenylate kinase AK1 gene knockout heart under metabolic stress. J. Biol. Chem. 275:41424-41429. http://dx.doi.org/10.1074/jbc.M007903200

Pucar, D., P. Bast, R.J. Gumina, L. Lim, C. Drahl, N. Juranic, S. Macura, E. Janssen, B. Wieringa, A. Terzic, and P.P. Dzeja. 2002. Adenylate kinase AK1 knockout heart: energetics and functional performance under ischemia-reperfusion. Am. J. Physiol. Heart Circ. Physiol. 283:H776-H782. http://dx.doi.org/10.1152/ajpheart.00116.2002

Renaud, O., P. Herbomel, and K. Kissa. 2011. Studying cell behavior in whole zebrafish embryos by confocal live imaging: application to hematopoietic stem cells. Nat. Protoc. 6:1897-1904. http://dx.doi.org/10 $.1038 /$ nprot.2011.408

Rissone, A., E. Foglia, L. Sangiorgio, S. Cermenati, S. Nicoli, S. Cimbro, M. Beltrame, F. Bussolino, F. Cotelli, and M. Arese. 2012. The synaptic proteins $\beta$-neurexin and neuroligin synergize with extracellular matrixbinding vascular endothelial growth factor a during zebrafish vascular development. Arterioscler. Thromb. Vasc. Biol. 32:1563-1572. http://dx.doi .org/10.1161/ATVBAHA.111.243006

Ryter, S.W., and A.M. Choi. 2002. Heme oxygenase-1: molecular mechanisms of gene expression in oxygen-related stress. Antioxid. Redox Signal. 4:625-632. http://dx.doi.org/10.1089/15230860260220120

Sauer, H., M. Wartenberg, and J. Hescheler. 2001. Reactive oxygen species as intracellular messengers during cell growth and differentiation. Cell. Physiol. Biochem. 11:173-186. http://dx.doi.org/10.1159/000047804

Shapiro, Y.E., M.A. Sinev, E.V. Sineva, V. Tugarinov, and E. Meirovitch. 2000. Backbone dynamics of Escherichia coli adenylate kinase at the extreme stages of the catalytic cycle studied by ${ }^{15} \mathrm{~N}$ NMR relaxation. Biochemistry. 39:6634-6644. http://dx.doi.org/10.1021/bi992076h

Sheehan, H.L., and G.W. Storey. 1947. An improved method of staining leucocyte granules with Sudan black B. J. Pathol. Bacteriol. 59:336-337. http://dx.doi.org/10.1002/path.1700590142 
Single, B., M. Leist, and P. Nicotera. 1998. Simultaneous release of adenylate kinase and cytochrome $\mathrm{c}$ in cell death. Cell Death Differ. 5:10011003. http://dx.doi.org/10.1038/sj.cdd.4400462

Sood, R., M.A. English, M. Jones, J. Mullikin, D.M. Wang, M. Anderson, D. Wu, S.C. Chandrasekharappa, J. Yu, J. Zhang, and P. Paul Liu. 2006. Methods for reverse genetic screening in zebrafish by resequencing and TILLING. Methods. 39:220-227. http://dx.doi.org/10.1016/j.ymeth.2006 .04 .012

Sood, R., B. Carrington, K. Bishop, M. Jones, A. Rissone, F. Candotti, S.C, Chandrasekharappa, and P. Liu. 2013. Efficient methods for targeted mutagenesis in zebrafish using zinc-finger nucleases: data from targeting of nine genes using CompoZr or CoDA ZFNs. PLoS ONE. 8:e57239. http://dx.doi.org/10.1371/journal.pone.0057239

Takubo, K., N. Goda, W. Yamada, H. Iriuchishima, E. Ikeda, Y. Kubota, H. Shima, R.S. Johnson, A. Hirao, M. Suematsu, and T. Suda. 2010. Regulation of the HIF-1alpha level is essential for hematopoietic stem cells. Cell Stem Cell. 7:391-402. http://dx.doi.org/10.1016/j.stem.2010 .06 .020
Thisse, C., and B. Thisse. 2008. High-resolution in situ hybridization to whole-mount zebrafish embryos. Nat. Protoc. 3:59-69. http://dx.doi .org/10.1038/nprot.2007.514

Traver, D., B.H. Paw, K.D. Poss, W.T. Penberthy, S. Lin, and L.I. Zon. 2003. Transplantation and in vivo imaging of multilineage engraftment in zebrafish bloodless mutants. Nat. Immunol. 4:1238-1246. http:// dx.doi.org/10.1038/ni1007

Warlich, E., J. Kuehle, T. Cantz, M.H. Brugman, T. Maetzig, M. Galla, A.A. Filipczyk, S. Halle, H. Klump, H.R. Schöler, et al. 2011. Lentiviral vector design and imaging approaches to visualize the early stages of cellular reprogramming. Mol. Ther. 19:782-789. http://dx.doi.org/10.1038/ mt.2010.314

Westerfield, M. 1995. The Zebrafish Book: A Guide for the Laboratory Use of Zebrafish (Danio rerio). Third edition. University of Oregon Press, Eugene, OR. 385 pp.

Whitford, P.C., O. Miyashita, Y. Levy, and J.N. Onuchic. 2007. Conformational transitions of adenylate kinase: switching by cracking. J. Mol. Biol. 366: 1661-1671. http://dx.doi.org/10.1016/j.jmb.2006.11.085 